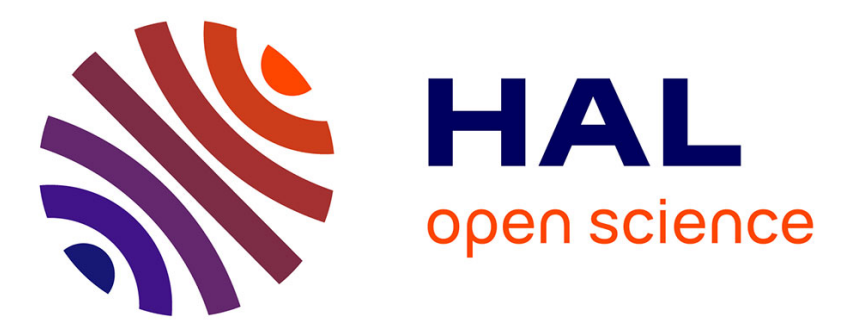

\title{
Une plaque émaillée celtique découverte à Paillart (Oise)
} Germaine Leman-Delerive, Jacques Bonte, Venceslas Kruta

\section{To cite this version:}

Germaine Leman-Delerive, Jacques Bonte, Venceslas Kruta. Une plaque émaillée celtique découverte à Paillart (Oise). Gallia - Fouilles et monuments archéologiques en France métropolitaine, 1986,44 (1), pp.29-53. 10.3406/galia.1986.2851 . hal-01940836

\section{HAL Id: hal-01940836 https://hal.science/hal-01940836}

Submitted on 27 Feb 2020

HAL is a multi-disciplinary open access archive for the deposit and dissemination of scientific research documents, whether they are published or not. The documents may come from teaching and research institutions in France or abroad, or from public or private research centers.
L'archive ouverte pluridisciplinaire $\mathbf{H A L}$, est destinée au dépôt et à la diffusion de documents scientifiques de niveau recherche, publiés ou non, émanant des établissements d'enseignement et de recherche français ou étrangers, des laboratoires publics ou privés.

\section{(이) $\$$}

Distributed under a Creative Commons Attribution - NonCommercial - NoDerivatives 44.0 


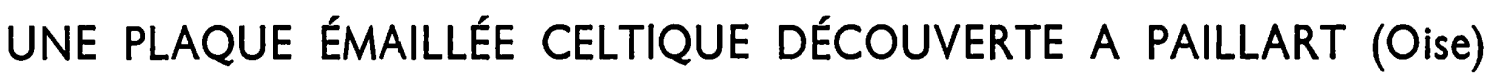

\author{
par Germaine LEMAN-DELERIVE \\ avec les contributions de Jacques BONTE ef Venceslas KRUTA
}

Une plaque de harnais au décor émaillé fut découverte fortuitement en 1970 à Paillart, au lieu-dit La Haule Bailly (fig. 1), lors de travaux agricoles. En dépit de son originalité sur le continent, elle est restée jusqu'à ce jour inédite. Pourtant son intérêt est doublement manifeste, d'une part à cause de la qualité de son exécution et de son excellent état de conservation, d'autre part à cause de sa provenance, tout à fait excentrique par rapport à l'aire habituelle des trouvailles de ce genre.

On ne connaît pas le contexte archéologique précis de cette plaque puisqu'elle fut ramassée en surface. Toutefois, son lieu de découverte a élé nolé avec certitude : parcelle 30 , section $\mathrm{W}$ de la commune de Paillart, et une fouille devrait être entreprise à cet endroit. Il est certain en effet que l'emplacement a connu une intense occupation aux $\mathrm{I}^{\mathrm{er}}$ et $\mathrm{II}^{\mathrm{e}}$ siècles de notre ère ; une prospection de surface laisse découvrir d'innombrables tessons de sigillée et de poteries communes. Le site a été également repéré par R. $\Lambda$ gache ${ }^{1}$ mais aucun plan détaillé de l'établissement n'est révélé par la photographie aérienne.

Le village de Paillart se trouve à $25 \mathrm{~km}$ environ au s. d'Amiens, à $4 \mathrm{~km}$ au $\mathrm{n}$. de Breteuil, dans le nord du département de l'Oise. Le lieu-dit La Haule Bailly, entre le chemin rural de Rome et celui de La Haule Bailly, situé sur le plateau qui domine d'une cinquantaine de mètres la vallée marécageuse de la Noye, petit affluent de la Somme, qui prend sa source un peu au s. de Vendeuil-Caply. Sur la rive opposée, la voie romaine Senlis-Amiens (chaussée Brunehaut) marque encore le paysage. Ce tracé antique, bien repéré au sol, a été rapproché de la voie attribuée à Agrippa par Strabon ${ }^{2}$, voie qui relie Lyon à Amiens et Boulogne-sur-Mer par Senlis ${ }^{3}$. A la sortie s. du village de Paillart, où la chaussée Brunehaut franchit la Noye, se greffe l'embranchement d'une route sans doute postérieure qui aboutit à Beauvais par Vendeuil-Caply. L'importance de ce dernier site mériterait assurément plus d'une mention : sur la colline des Chatelets (ou du Catelet) et dans ses environs immédiats se trouve une agglomération connue par ses quartiers d'habitat, deux théâtres, un camp romain daté de l'époque césarienne et, découvert plus récemment, un fanum dont la première implantation serait pré-romaine. Le Bois de Calmont, plus au s., est également un site fortifié attribuable peut-être à l'époque celtique ${ }^{4}$. Les monnaies gauloises recueillies à Vendeuil-Caply témoignent d'ailleurs de l'importance de l'occupation ancienne du site ${ }^{5}$. A Rouvroyles-Merles, à l'e. de la chaussée Brunehaut, R. Agache situe un autre sanctuaire d'époque romaine, dont, l'emplacement pourrait se justifier par la proximité de la frontière entre les cités des Bellovaques et des Ambiens ${ }^{6}$.

1 R. Agache el B. Breart, Allas d'archéologie aérienne de la Picardie. La Somme proto-historique el romaine, Amiens, 1975, feuille de Saint-Just.

2 Strabon, IV, 6, 11.

3 P. Leman, La voie du Léman à l'Océan, la branche orientale. Elat de la question el proposilions nouvelles, dans Caesarodunum, X, Tours, 1975, pp. 102-108; P. LEMan et Cl. Seillier, Fouilles de Boulogne-sur-Mer, dans Bull. de la Soc. des Anliquaires de France, 1978-79, séance du 13 décembre, pp. 139-148.
4 G. Dufour, Renconire avec le sile de Vendeuil-Caply (Oise), dans Cellicum, IX, Roanne, 1963, suppl. à Ogam, 1963, pp. 229-238; R. AGACIE, La Somme préromaine e! romaine, dans Mem. de la Soc. des Anliquaires de Picardie, XXIV, Amiens, 1978, pp. 412-414.

5 Simone Schens, Traile de numismalique cellique, II, La Gaule Belgique, Paris, 1977, passim.

6 R. Agache, op. cit., 1978, pp. 411-412. 
La plaque mesure $9,8 \mathrm{~cm}$ sur $10,5 \mathrm{~cm}$. De profil légèrement convexe, elle est en bronze coulé, orné d'ajours, de gravures et d'émail champlevé rouge et jaune. Le bronze a été également enduit d'une couverte transparente (fig. 2 et 3$)^{?}$.

Le décor est construit sur deux axes approximativement perpendiculaires, sur lesquels s'organisent et se combinent deux motifs, à savoir le triangle curviligne flanqué d'ocelles (qui peut être aussi considéré comme un motif en pelte) et une sorte de dérivé de la virgule ou spirale, tous deux réalisés en ajour, ou avec un remplissage d'émail, ou en simple gravure. L'artiste a aussi joué sur la dimension de ces unités décoratives : le couple triangles-ocelles est répété en trois échelles différentes; seules les ocelles de pâte jaune sont de grandeur à peu près constante. Par ailleurs, une autre caractéristique de ce bel objet est la symétrie par rabattement utilisée pour chaque élément décoratif. En réalité, cette symétrie est imparfaite : un examen attentif permet de vérifier qu'aucun des éléments n'est le décalque exact de son vis-à-vis.

Le centre de la plaque est constitué de deux triangles curvilignes s'opposant par la pointe et traités en ajour. De part et d'autre des deux exlrémités du triangle se placent deux ocelles comblés d'émail jaune. Un tracé gravé dans le bronze encadre chaque triangle ajouré, de telle sorte que cette parlie centrale se présente comme deux peltes opposées dont les pieds sont également flanqués de triangles curvilignes, réalisés en ajour, de taille plus réduite. L'association de ces petils triangles avec les ocelles de pâte jaune évoque également deux peltes orientées perpendiculairement.

Le même schéma se répète dans les deux lobes latéraux construits sur le plus grand axe passant par le sommet des petits triangles centraux. Seules diffèrent dans cette zone la dimension des grands triangles, légèrement plus petits, et leur réalisation en émail rouge. Toutefois, chaque partie extérieure du motif quadruple se prolonge en se combinant avec le deuxième élément décoratif, à savoir la virgule. Un trait gravé, assez irrégulier, qui prend naissance, soit dans la périphérie du triangle ajouré, soit à la circonférence d'un ocelle (sur l'autre lobe), vient s'enchaîner autour du second ocelle, dessinant ainsi une sorte de tête d'oiseau fantastique. Enfin, toujours sur ce même axe, la liaison entre le groupe central et les lobes latéraux est faite à nouveau par un triangle curviligne, légèrement différent par son remplissage de pointillés et par l'orientation de sa pointe vers l'extérieur.

Sur le plus petit axe, les deux autres lobes n'introduisent pas de nouveaux éléments décoralifs. La dimension du triangle ajouré et le traitement de zones en pointillés modifient légèrement la présentation de ces deux parties. L'émail rouge semble jouer un rôle décoratif plus défini que dans les lobes centraux, car on peut retrouver à nouveau le triangle curviligne à la base des doubles ocelles avec lesquels il vient également se combiner en une nouvelle pelte.

Autour des trois ensembles du grand axe, court une ligne de pointillés, au tracé maladroit, et d'ailleurs corrigé à un endroit, qui affecte un profil réniforme.

Les angles externes, situés entre chaque lobe, sont occupés par une virgule spiralée, dont le centre est comblé de pointillés, et dont l'aboutissement externe dessine le bord de la plaque.

Les différents motifs ont été construits au compas, à la manière reconnue sur les miroirs, sans doute contemporains ${ }^{8}$. La technique de fabrication de l'émail a été étudiée par J. Bonte (voir les analyses en Annexe I). En particulier, la coloration verte du bronze, qui s'harmonise si heureusement avec les émaux rouge et jaune, s'explique par une couverte transparente qui aurait pu recouvrir à l'origine tout le droit de la pièce, et qui a débordé légèrement sur le revers. L'émail jaune s'est remarquablement conservé, à l'exception d'un ocelle vidé de son contenu, tandis que l'émail rouge s'est craquelé en plusieurs endroits.

7 On gardera ici le terme traditionnel d'émail comme le proposent J. L. Davies et M. G. Spratling, The Seven Sislers Hoard: a centenary study, dans Welsh Antiquity (ed. G. C. Boon et J. M. Lewis), Cardiff, 1976, pp. 121-147, cf. p. 145 , note 38 ; contra: M. J. IIUGHEs, $A$ technical study of opaque red glass of the Iron in Brilain, dans Proceedings of the Prehistoric Society, vol. 38, 1972, pp. 37-107, cf. p. 98-99.

8 P. R. Lowery, R. D. A. Savage, R. L. Wilkins, A Technical Study of the Designs on the British Mirror Series, dans Archaeologia, 105, 1976, pp. 99-126. 


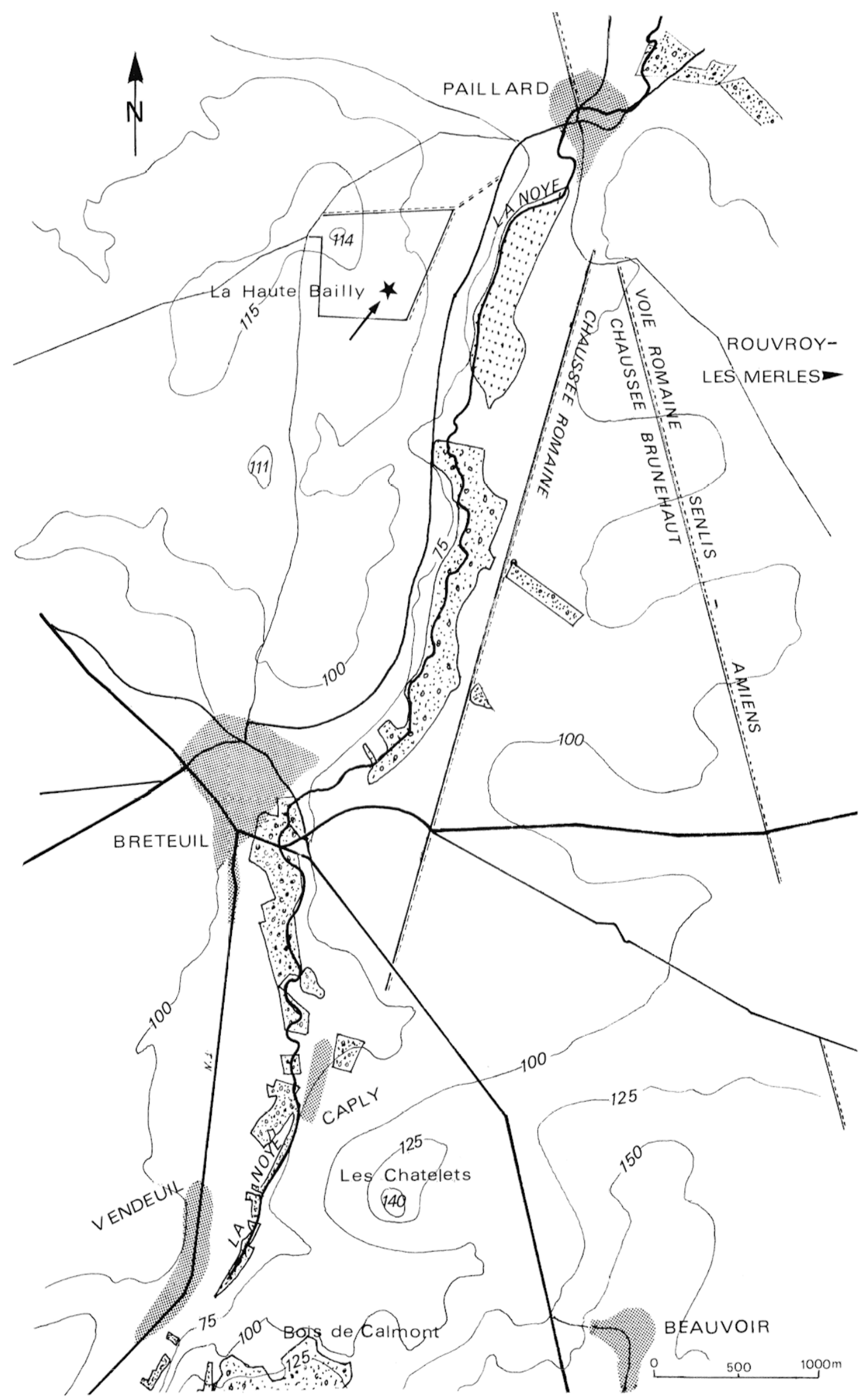

1 Plan de localisation de la découverte; en haut, au centre flèche et étoile. 


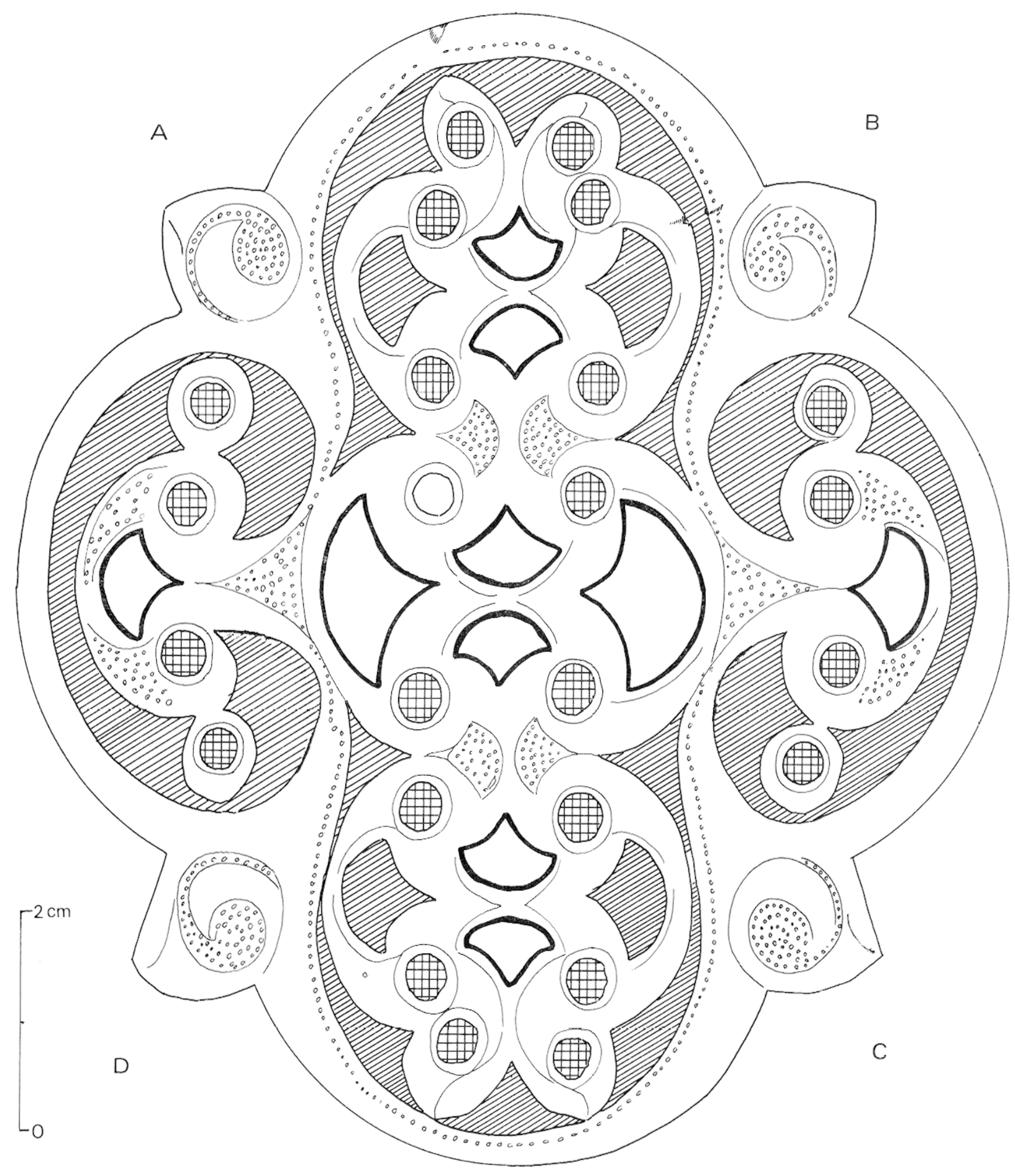

2 Plaque de Paillard. Relevé schémalique du décor. 


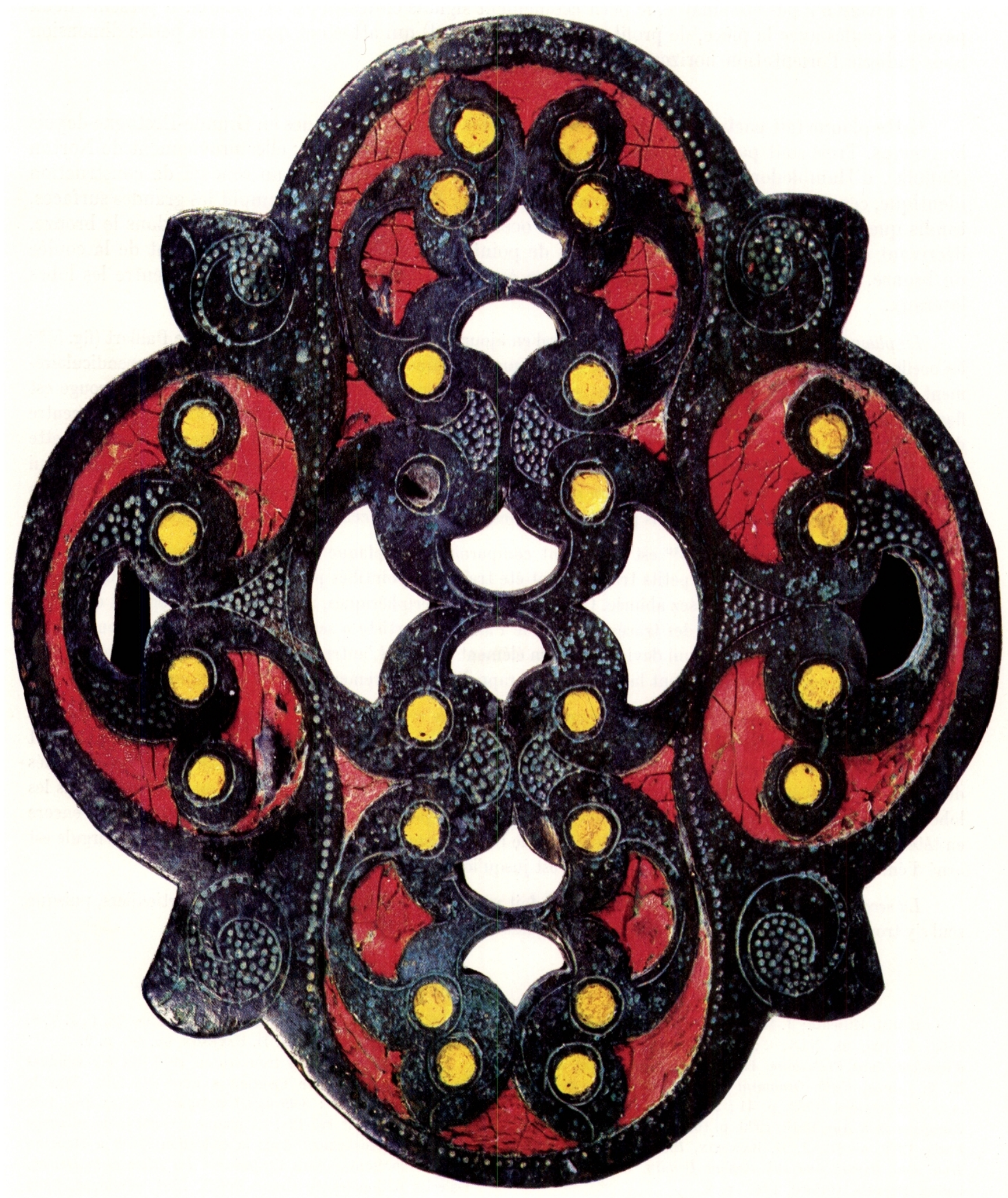

3 Plaque de Paillard. 
Le revers n'a pas été émaillé, le petit écoulement signalé ci-dessus est accidentel. Il présente deux passants coulés avec la pièce, de profil rectangulaire (fig. 4) qui attestent que la plus petite dimension nous indique l'orientation horizontale de l'objet.

Cette plaque fait partic d'une petite série de pièces analogues, connues en Grande-Bretagne depuis longtemps. Trois sont particulièrement proches de l'exemplaire français : elles proviennent de Norton (Suffolk), d'Hambledon (South Buckinghamshire) et de Londres. Outre un schéma de construction jdentique, ces quatre plaques ont en commun l'emploi de l'émail rouge qui remplit les grandes surfaces, tandis que la pâte jaune occupe seulement les ocelles. Interviennent aussi la gravure dans le bronze, décrivant des triangles curvilignes, comblés de pointillés, et l'ajour, réalisé au moment de la coulée du bronze. Le motif en virgule spiralée occupe également chaque espace intercalaire entre les lobes latéraux.

La plaque de Londres présente un motif central en ajour qui rappelle de très près l'objet de Paillart (fig. 5$)^{9}$; les ocelles de pâte jaune ont disparu, laissant une plaque plus importante aux triangles orientés perpendiculairement. L'ornementation des quatre lobes est simplifiée : d'une part, un triangle curviligne en émail rouge est flanqué d'ocelles jaunes : il est vraisemblable, à en juger par le tracé extérieur, que l'ocelle externe soit le centre d'une virgule spiralée, mais la mauvaise conservation de l'objet ne permet pas de lire aisément le décor de cette zone. Faisant la liaison avec le centre, se retrouve un couple de triangles comblés de pointillés, composition qui rappelle de très près celle de Paillart. L'autre partie est occupée par un tracé en accolade ponctué de trois points d'émail jaune, qui se situent, du moins pour les exemples extérieurs, au centre d'un motif en spirale.

La pièce de Norton (Suffolk) ${ }^{10}$ est également comparable à la plaque de Paillarl par son décor central, à la différence près que les deux petits triangles ont été traités en pointillés plutôt qu'évidés (fig. 6). Cette zone centrale est malheureusement assez abîmée. Quant aux parties périphériques, il est tout à fait frappant que cette plaque constitue la moitié d'un des tracés de celle de Paillart. L'artiste a seulement remplacé un ajour par de l'émail rouge, el agrandi l'autre, qui devient ainsi un élément central. L'autre lobe doit être rapproché plus précisément de la plaque de Londres, dont la composition rappelle singulièrement celle de la plaque française.

Une des deux plaques d'Hambledon (Dorset), découvertes récemment ${ }^{11}$, est d'une conception légèrement différente : le motif central, en triangle curviligne, traité en ajour, a été agrandi, si bien qu'on pourrait plutôt attribuer à cet objet une construction bilobće, et non quadrilobée (fig. 7, 1 et $1^{\prime}$ ). Les triangles évidés sont bordés de zones en pointillés qui affectent une forme approchée de l'éventail. Le décor coloré s'est développé dans les lobes latéraux : on y retrouve le triangle curviligne, les ocelles jaunes au centre de motifs en spirale, ou encore en tête d'oiseau, qui évoquent de très près le dessin de l'objet français. Seule différence, le motif de la virgule est orné d'émail rouge, alors que sous cette forme, il est jusqu'ici gravé.

La seconde plaque d'Hambledon (fig. 7, 2 et 2') fait partie d'un ensemble aux caractères particuliers, puisque seul s'y trouve l'émail rouge el que les motifs sont également différents ${ }^{12}$.

9 British Muscum; J.-M. KEмble, Horae Ferales, I.ondon, 1863, p. 195, pl. XIX, 2; Françoise HsinY, Emailleurs d'occident, dans Préhistoire, II, 1933, pp. 66-146, fig. 14, I ; E. T. Lrens, Cellic Ornament in the British Isles down to A.D. 700, Oxford, 1933, p. 41 ; J. V. S. MlGaw, Art of the European Iron Age, Bath, 1970, p. 163 ; V. Kruta, Les Celtes, Paris, 1978, no 81 ; J. D. BATssox, Enamel-working in the Iron Age, Roman and sub Roman Brilain, British Archeological Reports, Oxford, 1981, p. 9.

10 Moyse Hall Museum, Bury St Edmunds; J. M. Kғmble, op. cit., pl. XIX, 4 ; R. Clarke, The Iron Age in Norfolk and Suffolk, dans Archaeological Journal, XGVI, 1939, p. 1-113, cf. p. 69 ; Françoise HiNkY, op. cit., fig. 20, 4 ; J. V.S. Migaw, op. cit., p. 163 ; J. D. Batrson, op. cit., p. 9.

11 Les deux plaques trouvées en 1978 ont été venducs en 1983 : la première mention a donc été faite dans le catalogue de vente : Solheby's Calalogue of Antiquities, juin 1983 , p. 35-36, no 127-128. La plaque à émail jaune et rouge se trouve acluellement dans la collection Menil à Houston et a été présentéc dans l'exposition La Rime el la Raison, Galeries nationales du Grand Palais, 1984, catalogue, Paris 1984 , p. 321 , no 95 .

12 Son lieu de conservation actuel ne semble pas connu. 

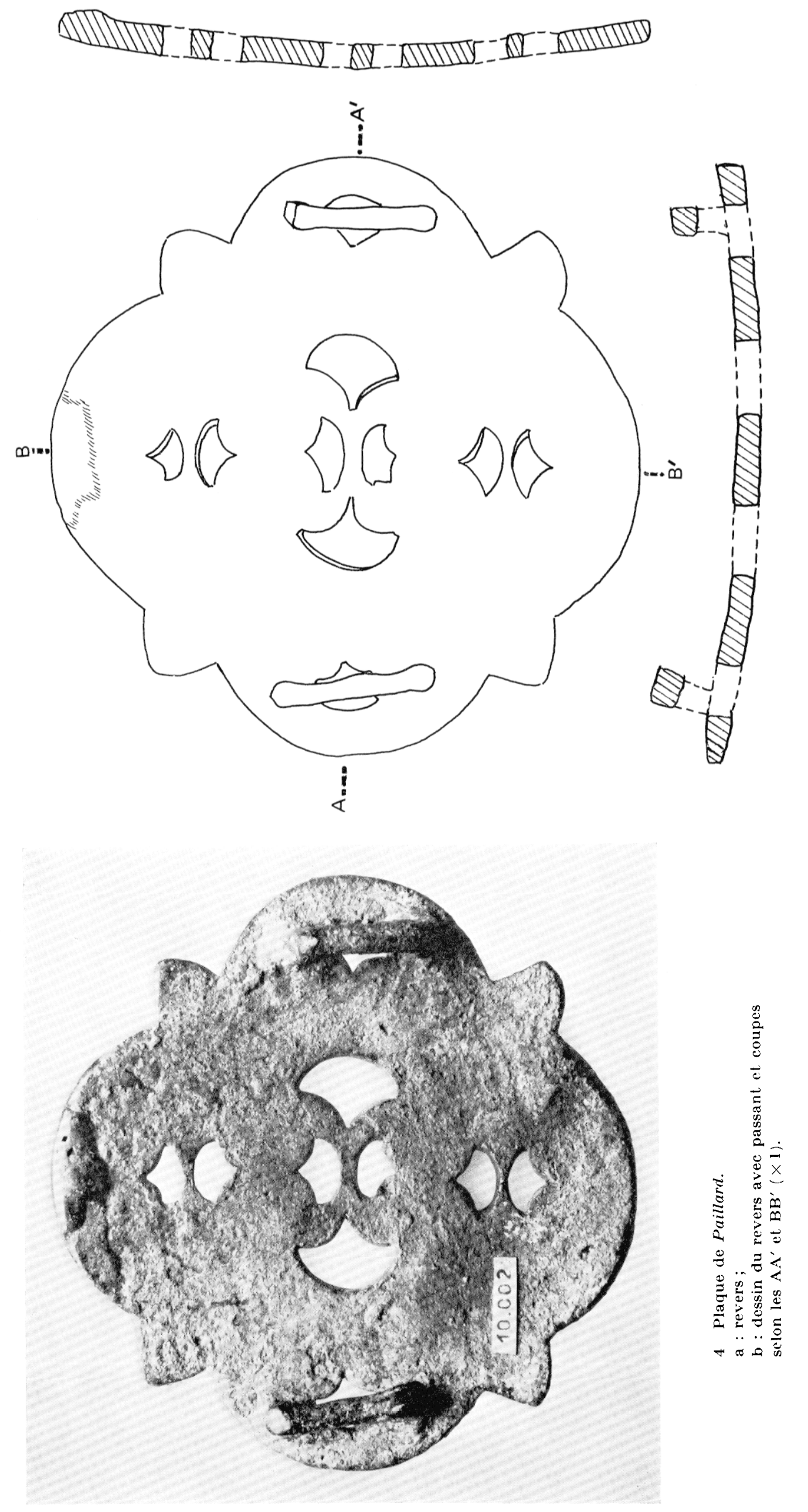


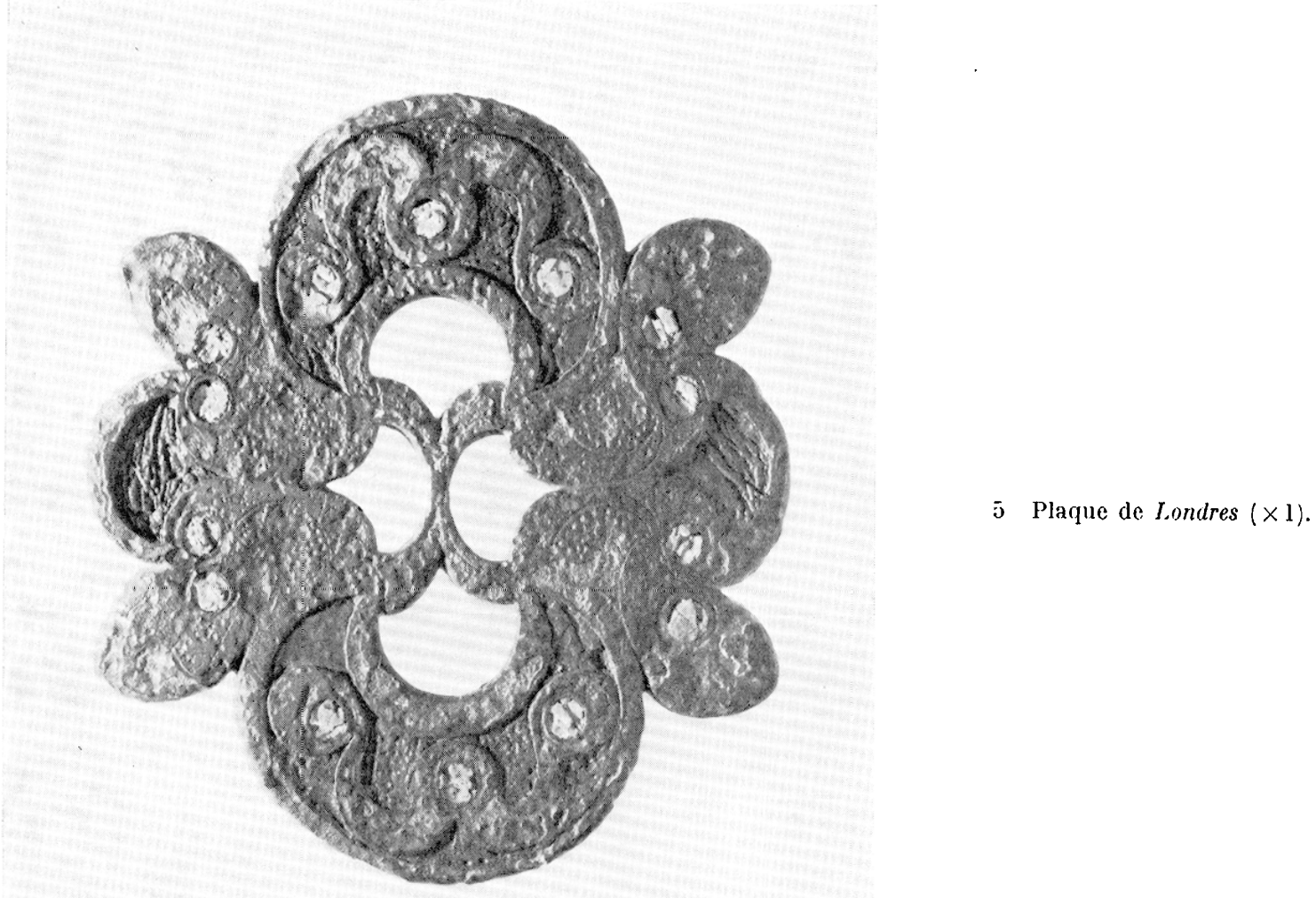

6 Plaque de Norton $(\times 1)$.

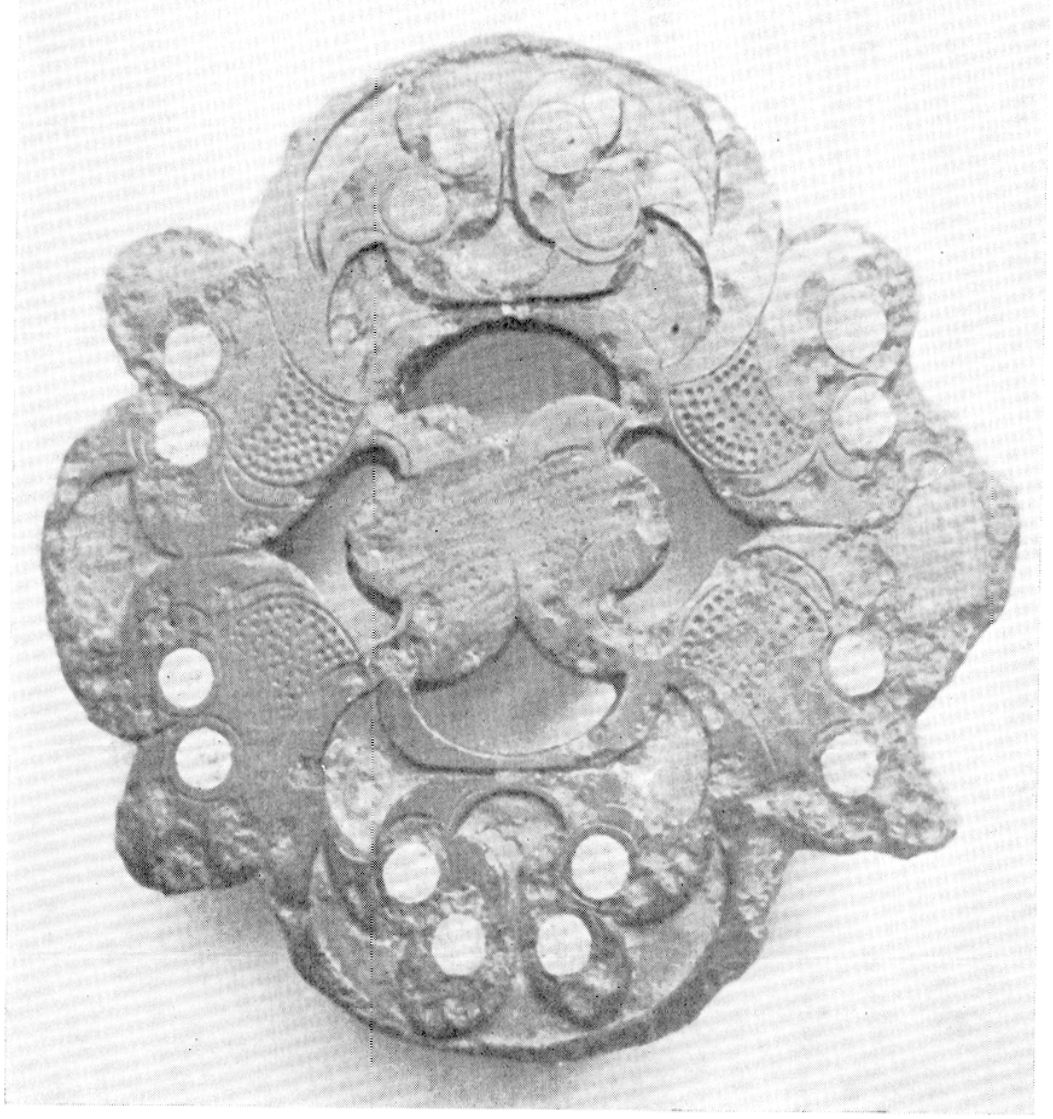



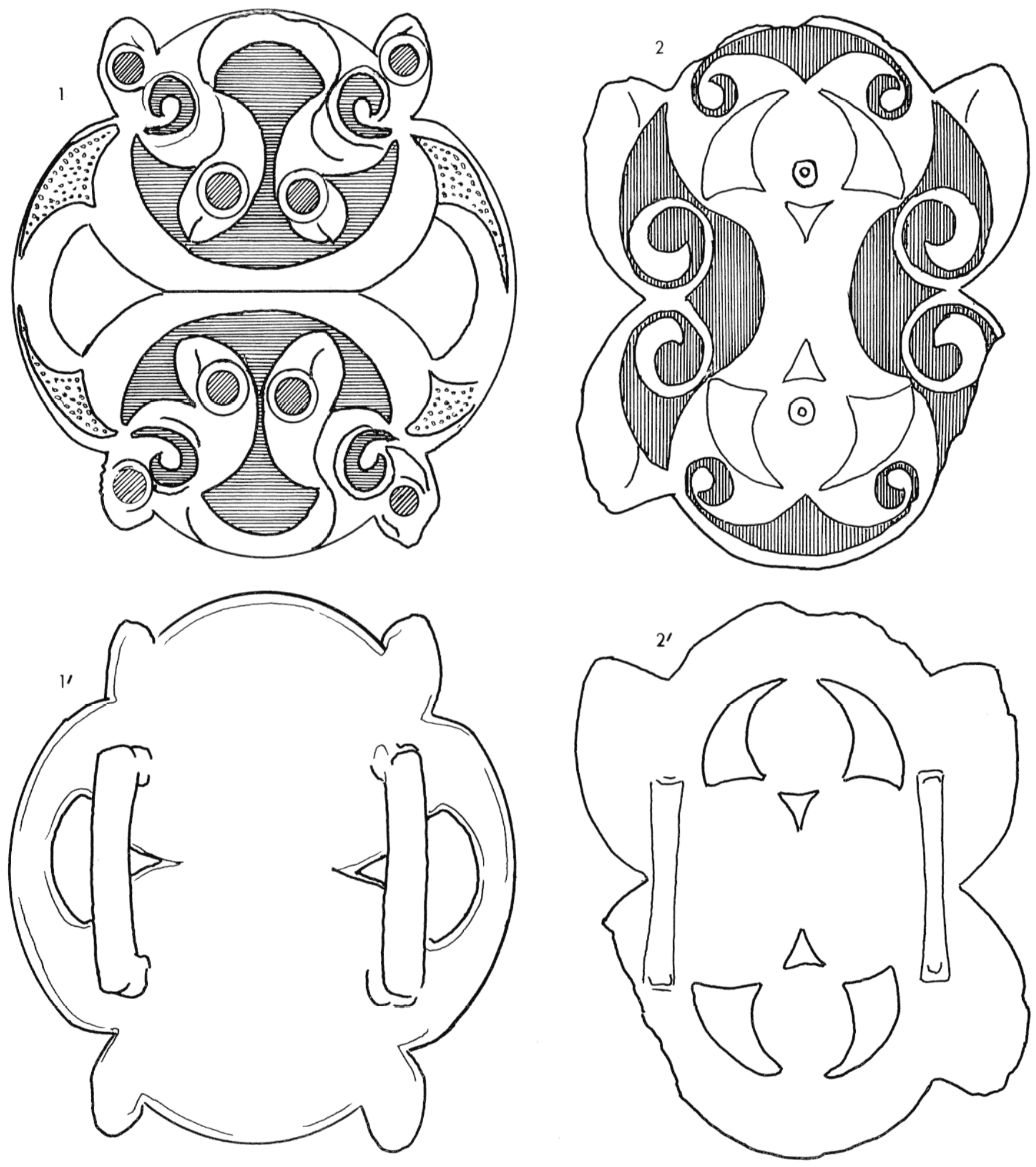

71 et $1^{\prime}:$ Plaque d'Hambledon I (collection Menil à Houston (Texas)). 1 ( $\times 1$ ). 2 et 2': Plaque d'Hambledon II (collection particulière) $2^{\prime}(\times 1)$. 

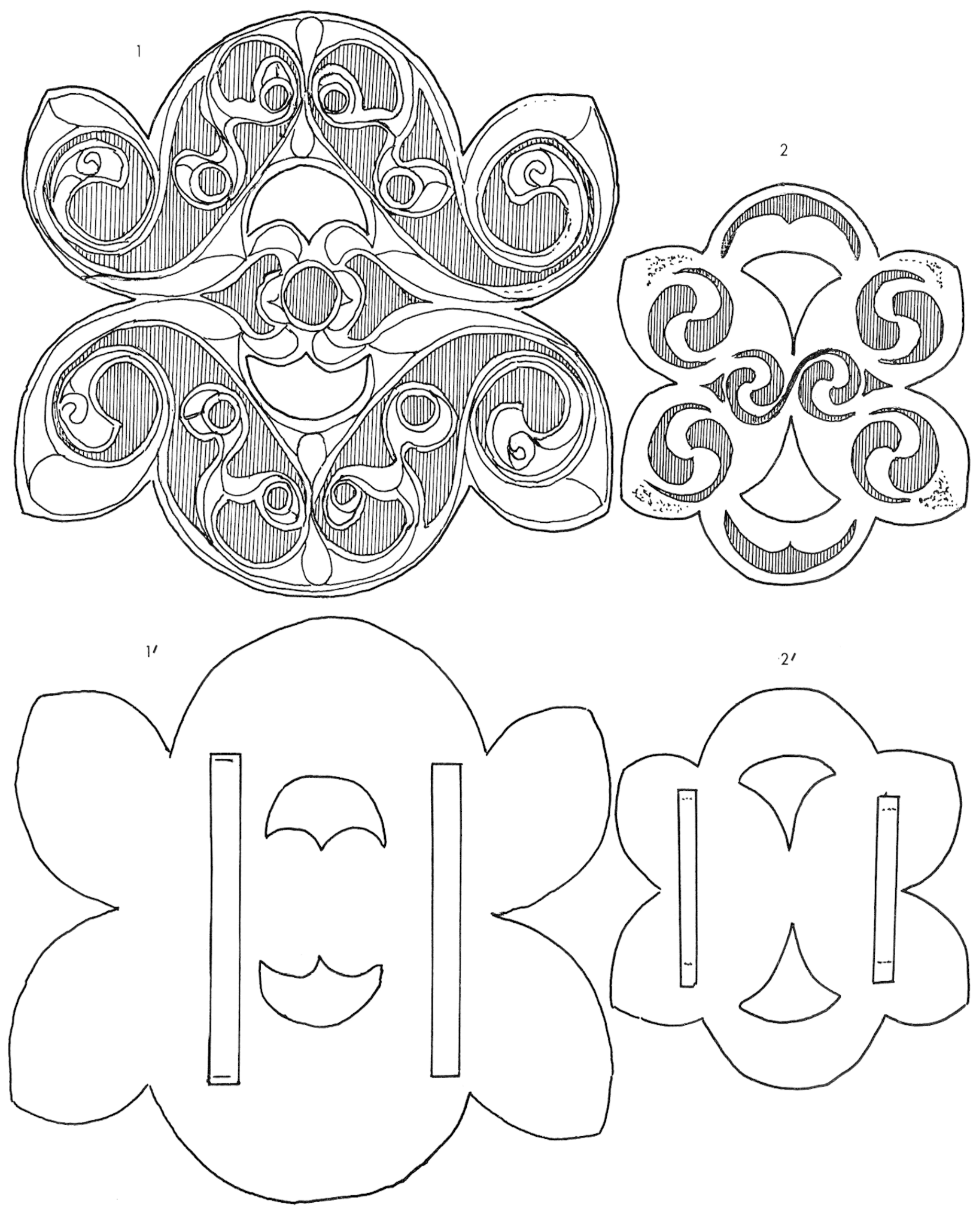

81 et $1^{\prime}$ : Plaque de Santon I, d'après Mansel G. Spratling $(\times 1)$. 2 et $2^{\prime}$ : Plaque de Santon II, d'après Mansel G. Spratling $\left.i \times 1\right)$. 

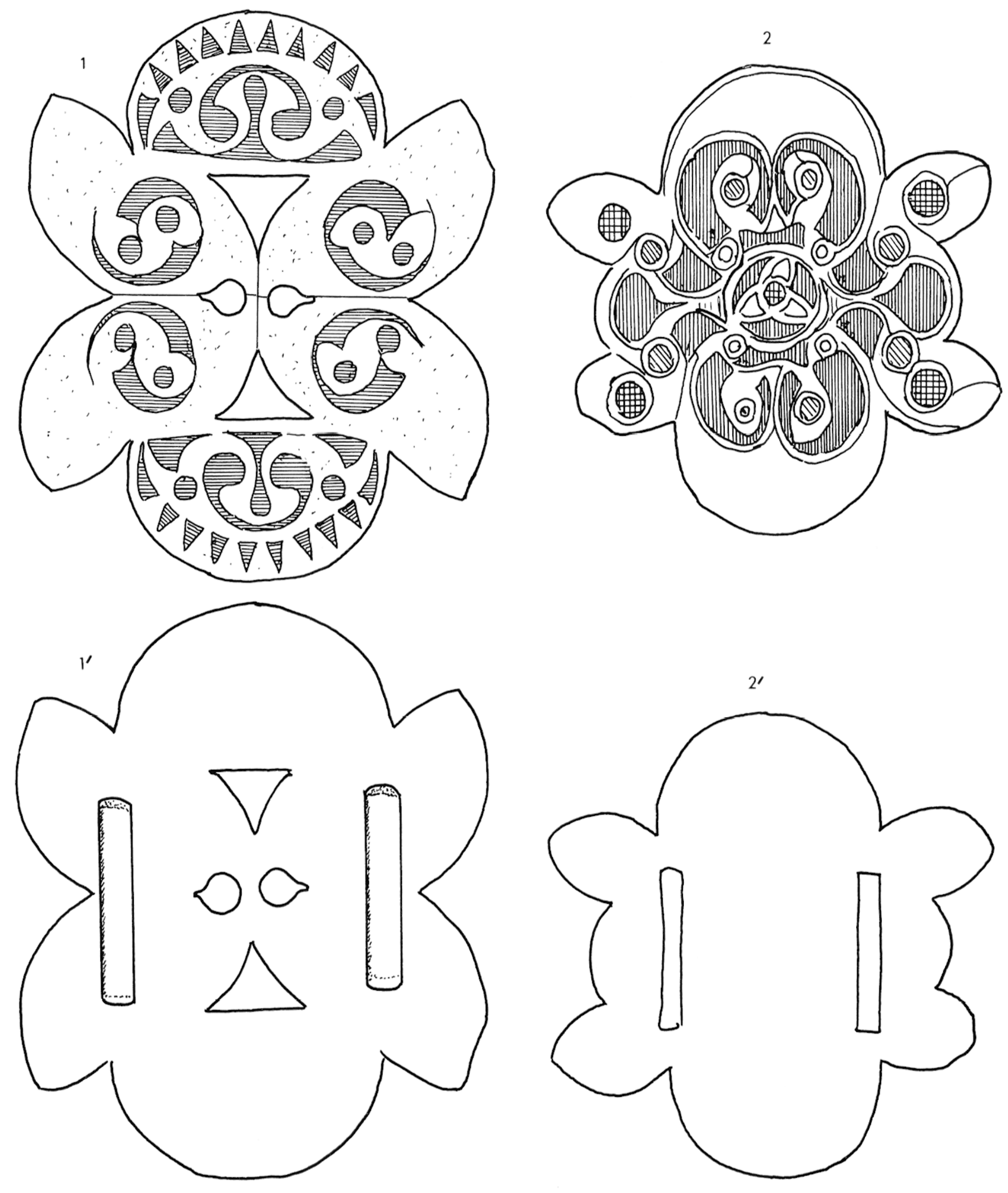

91 et $1^{\prime}$ : Plaqque de Polden Hill, d'après Mansel G. Spratling $(\times 1) .2$ et $2^{\prime}:$ Plaque de Westhal, d'après Mansel G. Spratling $(\times 1)$. 
Les deux pièces les plus exceptionnelles de cette catégorie proviennent de Santon (Norfolk) (fig. 8) et leur qualité d'exécution n'a d'égal que leur excellent état de conservation ${ }^{13}$. L'une, la plus décorée, est élaborée à partir d'un schéma d'accolade double dont les extrémités se terminent en enroulement (fig. 8, 1 et $1^{\prime}$ ). Dans l'espace ainsi délimité, Majolie Lenerz de Wilde voit un masque pathétique, typique du style dit de "Cheshire Cat " ${ }^{\mathbb{4}}$. Sur le plan technique, l'émail rouge est traité comme un fond, tandis que la gravure à la finesse exceptionnelle a dû vraisemblablement être réalisée en fin d'exécution et non avant la coulée du bronze. Le motif de l'ocelle (ici en matière rouge), au centre d'un tracé spiralé, répété d'ailleurs de façon concentrique aux extrémités, est utilisé à la manière constatée aussi sur la plaque de Isondres. L'autre plaque de Santon, moins décorée (fig. 8,2 et $2^{\prime}$ ), rappelle quelque peu l'objet d'Hambledon par son motif central.

La plaque de Polden Hill (Somerset) (fig. 9, 1 et $1^{\prime}$ ) trahit une sorle de raideur dans la composition, en raison peut-être des larges surfaces non décorées ${ }^{15}$. Un motif est inhabituel : la frise de triangles qui souligne la courbure d'un des lobes ; par ailleurs, la disposition des doubles ocelles - ici en émail rouge - conduil à distinguer plus facilement une tête d'oiseau qu'un motif spiralé. Ce dessin se retrouve aussi sur l'autre plaque émaillée, en forme de palmette, issue du même lot d'objets.

Les trouvailles de Westhall (Suffolk) présentent également des analogies avec les objets de Londres, de Norton et de Paillart, notamment par l'utilisation du motif en triangle flanqué d'ocelles en pâte colorée, et de la virgule ou spirale (fig. 9, 2 et $2^{\prime}$ ). Toutefois, la partie centrale de la plaque est traitée différemment, le motif ajouré est remplacé par une sorte de triscèle. De plus, si les parties largement creusées sont remplies d'émail rouge, les ocelles ont l'aspect de l'ivoire, mais sont aussi décrits comme de l'émail bleu. Én réalité, la pâte blanche et opaque semble bien provenir d'une décomposition de l'émail bleu ${ }^{16}$.

Enfin, si les comparaisons portent sur les revers, il convient de souligner la taille plus réduite des passants de la plaque de Paillart.

Les similitudes de décor observées entre ces pièces britanniques ${ }^{17}$ et l'objet français amènent à attribuer à celui-ci une origine insulaire (fig. 10). Bien plus, il est même permis de se demander si les plaques de Paillart, de Londres, de Norton et d'Hambledon ne proviennent pas du même atelier. Il n'est pas impossible, en effet, que l'exemplaire unique trouvé en France constitue le prototype des pièces apparemment moins complexes découvertes en Grandc-Bretagne. En outre, cettc pièce tranche sur toutes les autres à la fois par sa grandeur et par la beauté de son décor. Seule une analyse des composants (bronze et émaux) pourrait confirmer cette hypothèse ${ }^{18}$.

Malheureusement, les trouvailles anglaises n'ont pas de contexte chronologique précis. Seuls les objets de Polden Hill, Santon et Westhall ont été trouvés en dépôt. Lc lot de Polden Hill, qui a fait l'objet d'une publication récente, est placé au milicu de deux fibules caractéristiques de cette époque ${ }^{19}$.

13 Musée d'Ethnologie el d'Archéologie de l'Universilé de Cambridge; Françoise Henry, op. cit., fig. 12, 4 ; R. Clarke, op. cit., p. 69 ; J. V. S. MEgaw, op. cil., p. 163, pl. VIII a ; P.-M. Duval, Les Celtes, Paris, 1977, fig. 225 ; J. D. Bateson, op. cit., p. 15-16.

14 M. Lenerz DE Win.mi, Le "style de Cheshire Cat". Un phénomène caractéristique de l'art celtique, dans L'arl celtique de la période d'expansion, IV el III' siècles avant notre ère, éd. P.-M. Duvai. et V. Krita, Genève-Paris, 1982, pp. 101-114, cf. p. 105, fig. 26.

15 British Museum; Françoise Hexry, op. cit., 12, 3; J. V. S. Megaw, op. cit., p. 163 ; J. W. Brailsford, The Polden Hill Hoard, Somerset, dans Proceedings of the Prehistoric Society, vol. 41, 1975, pp. 222-254, cf. pl. XXIII a ; J. D. Bateson, op. cit., p. 18.

16 British Museum; J. M. Kemble, op. cil., pl. XIX, 1 ; R. Clarke, op. cit., p. 68, pl. XVII, I-3; Françoise IIFnry, op. cil., fig. 16,2 ; E. T. L.eeds, op. cil., pl. I, 6, p. 41 ; J. V. S. MEgaw, op. cil., p. 163 ; J. D. Bateson, op. cil., p. 18. 17 L'utilisation du motif spiralé associé à la pelte flanquée d'ocelles se retrouve sur des plaques cruciformes : voir par exemple l'objet de provenance inconnue conservé au British Muscum : Françoise Henry, op. cit., fig. 20,3. Une autre pièce, trouvée à Canterbury, utilise également le motif spiralé : Françoise Hexry, op. cil., fig. 11,4; E. T. I.kEds, op. cit., p. 52, pl. II, 3 ; J. D. Bareson, op. cit., p. 9.

18 Les résultats d'analyses faites sur les objets du Brilish Muscum (voir M. J. Hughes, op. cit.) ne peuvent pas être comparés en raison des différences de méthodes employées. Pour J. V. S. Megaw, l'atelier ne peut que se situer dans l'Est de l'Angleterre, mais il est difficile de préciser davantage celte localisation.

19 J. W. Brailsford, op. cil., p. 232-3. 

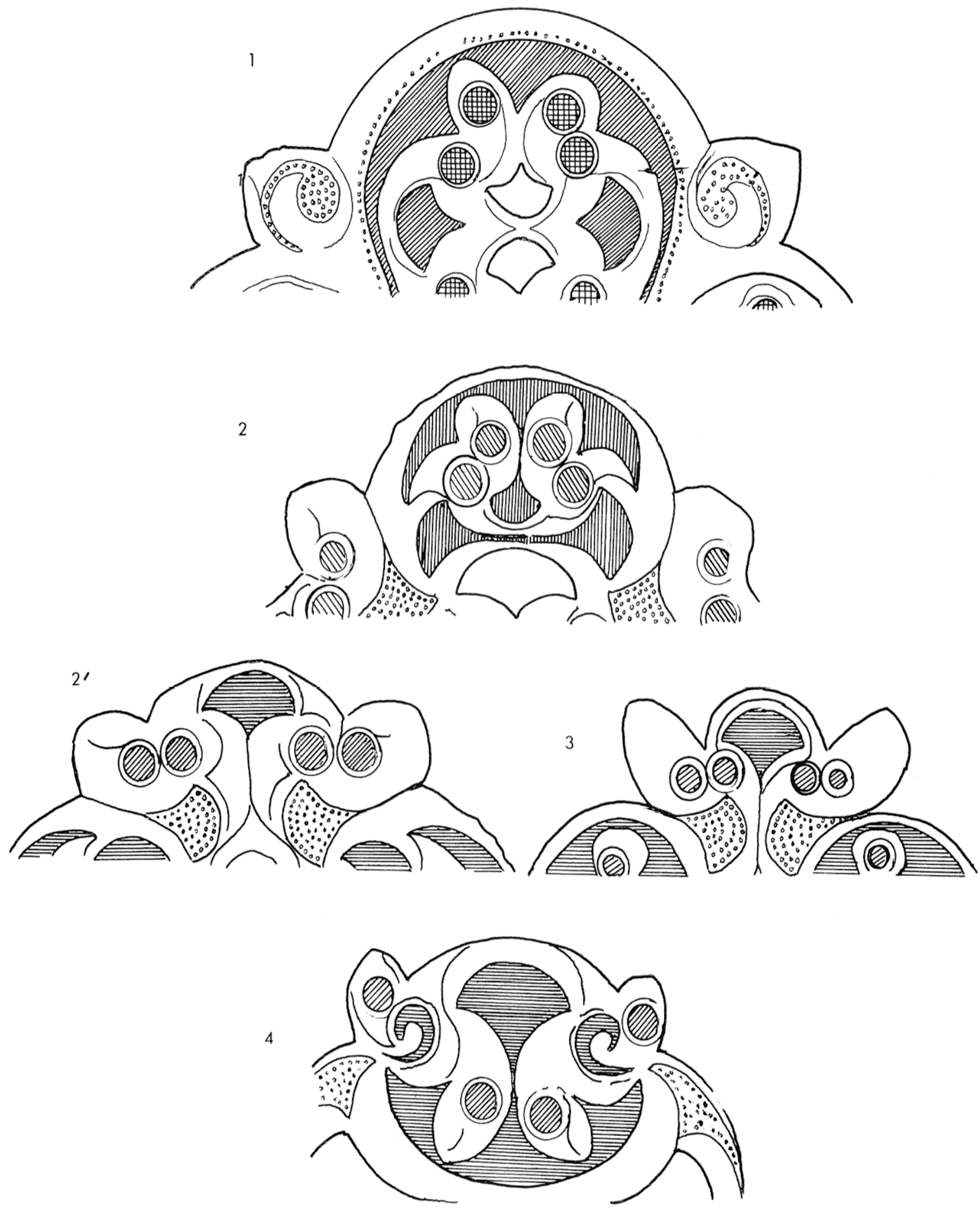

10 Schéma de comparaison entre les plaques : 1 - Paillard ; 2 - Norton, représentation de la partie supérieure ; 2 deuxième moitié perpendiculaire à la partie supérieure ; 3 - Londres ; 4 - Hambledon. 

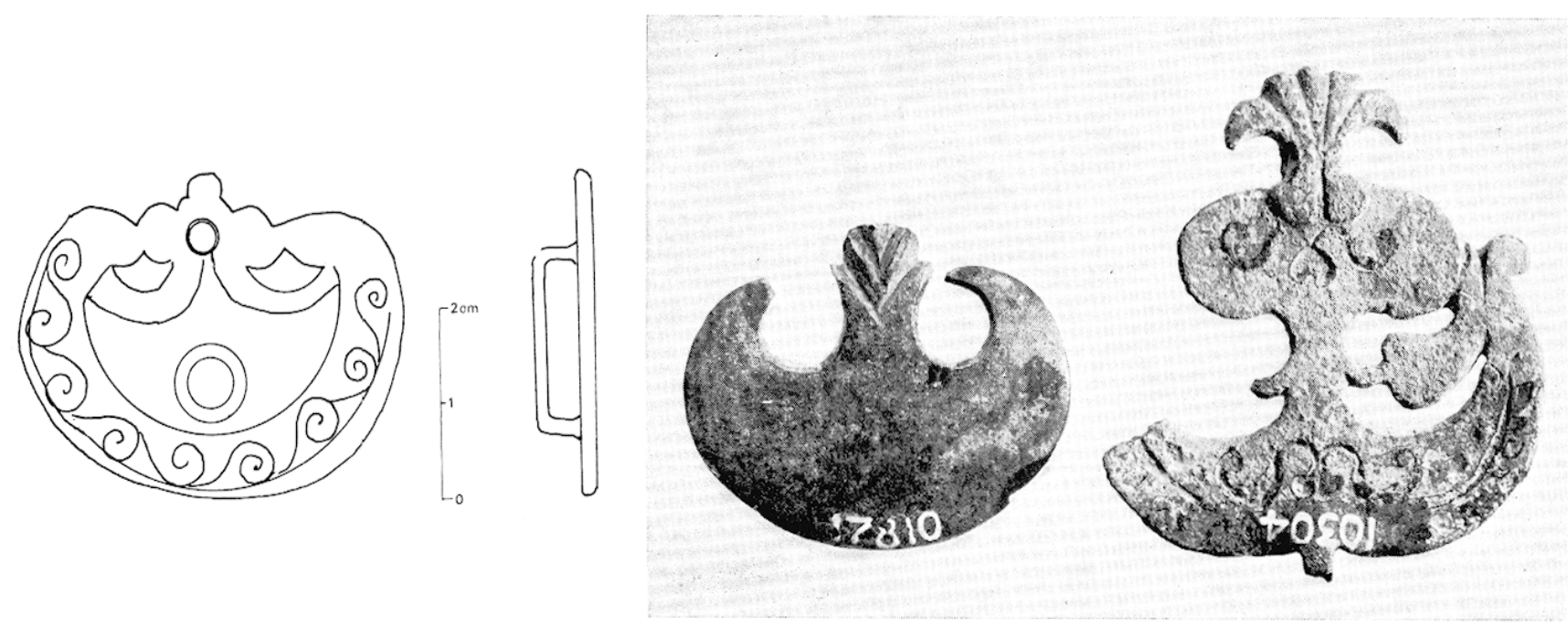

11 Plaque de Bailleul-sur-Thérain (dessin reconstitué, vue de face et vue de profil).

12 Petits bronzes de Trèves.

Les pièces de Santon doivent leur excellent état de conservation à leur dépôt dans un chaudron de bronze, en association avec des fibules datées également de 40 à 60 . Le trésor de Westhall, qui contenait une lampe du troisième quart du $\mathrm{i}^{\mathrm{er}}$ siècle, est mis en relation avec la révolte de Boudicca. Il est donc vraisemblable que la plaque de Paillart date également du milieu du I $^{\text {er }}$ siècle. L'absence de tout contexte ne permet toutefois pas d'inférer la date de son importation ou de son utilisation sur le continent. Son excellent état de conservation semble exclure une longue utilisation, mais il faut se garder en cette matière d'unc proposition trop précise.

Il est tentant d'évoquer la relation de ces pièces de harnachement celtique avec lcurs similaires d'époque romaine. Il ne saurait être question de traiter ici d'un sujet aussi vaste mais il convient néanmoins de rappeler une pièce découverte au siècle dernier, également dans l'Oise, à Bailleul-surThérain ${ }^{20}$. Cet objet, qui, malheureusement, disparut dans l'incendie du muséc de Beauvais en 1940 , est en forme de pelte, avec une frise de spirales sur le pourtour; deux triangles curvilignes ajourés allègent la partie supérieure, et deux cercles concentriques occupent la zone médiane (fig. 11). En dimension, cette pièce correspond à un lobe de la plaque celtique, celui qui précisément porte le motif en pelte. La frise spiralée inscrite dans une forme en croissant rappelle étrangement un certain type d'anneaux de harnachement d'origine britannique ${ }^{21}$. Deux passants de grande dimension se trouvent

20 A. G. Hountgant, Description des objets d'anliquiles locales, celtiques, gallo-romaines et mérovingiennes renfermés dans le cabinet de M. Houbigant, de Nogent les Vierges, dans Mémoires de la Société Archéologique de l'Oise, 1860, p. 409. 534 ; E. Worlliez, Allas des Antiquités celtiques, galloromaines el des monuments du moyen âge inédits ou peu connus du département de l'Oise, 1, 1867 (Manuscrit de la Société académique d'archéologie, des sciences et des arts du département de l'Oise. Bibliothèque municipale de Beauvais);
J.-C: BLAxchet, Les premiers métallurgistes en Picardie el dans le Nord de la France, dans Mémoires de la Societé Préhislorique Française, 17, 1984, p. 15.

21 R. Cl.Arke, op. cil., pl. XVII (trésor de Westhall), ou Françoise IIExry, op. cit., fig. 22, 2 (anneau provenant du Fayoum); l'origine de ce molif semble très proche du graphisme spiralé ou en tête d'oiseau des plaques du type Paillart : voir l'exemple donné par un anneau trouvé dans la Tamişe à Runnymède par Françoise Henry, op. cit., fig. 14, 2. 
à l'avers. Il est vraisemblable que cette plaque était émaillée, comme une autre, disparue également, qui fut trouvée par A. Terninck à Bétricourt (Pas-de-Calais) dans un cimetière du Bas-Empire ${ }^{22}$. Sur cet objet, la zone centrale est ornée d'émail bleu. Le dessin ne permet pas de discerner toutefois si cette dernière pièce présentait deux passants rectangulaires à l'avers, comme l'objet de Bailleul-sur-Thérain, ou encore de Sanxay (Vienne) ${ }^{23}$, ou deux rivets. En effet, on rencontre assez fréquemment, en particulier dans les niveaux du Bas-Empire, et en contexte militaire, de petits bronzes en forme de pelte plus ou moins simplifiée, qui se fixent par un système de rivets ${ }^{24}$ (cf. fig. 12).

II n'est peut-être pas inutile de rappeler combien tous les aspects matériels des différents échanges entre le continent et la Bretagne, au début de notre ère, sont loin d'être connus. Certes, des travaux ont attiré l'attention sur des échanges fréquents, sinon journaliers, d'objets domestiques commercialisés entre des régions portées naturellement à de telles relations, dont Strabon fait d'ailleurs mention ${ }^{25}$. I'autres pièces sont exceptionnelles par leur qualité et leur lieu de découverte en dehors des zones de contact obligé. La plaque trouvée à Ober Olm, dans la Hesse, sans conteste semble-t-il, appartient à une série d'objets proches des plaques quadrilobées. Le décor de triangles curvilignes, traité en ajour, ou en émail, évoque assurément les plaques de Norton, Westhall ou Paillart; la frise de triangles rappelle le motif de la pièce de Polden Hill mais il est plus difficile de trouver des éléments de comparaison aux bossettes centrale et latérale (fig. 13) ${ }^{26}$. A l'inverse, l'origine insulaire du miroir de Nimègue, au décor gravé exceptionnel, est incontestable. Selon l'interprétation couramment admise, le cheminement de cet objet s'expliquerait par le retour d'un auxiliaire batave de l'armée romaine de Bretagne ${ }^{27}$. L'autre pièce typiquement britannique est le bol de bronze de Leg Pickarski (Pologne), muni d'un bec verseur zoomorphe, particulièrement caractéristique de l'art des Celtes insulaires ${ }^{28}$. Ces régions de Germanie libre attestent des contacts avec le monde romain, et à travers celui-ci avec l'artisanat celto-britannique. Récemment publiée, une plaquette a été trouvée naguère à Bentumersiel, à l'embouchure de l'Ems ${ }^{29}$. Comme la plaque d'Ober Olm, elle est très abîmée; on a pu y voir toutefois un décor émaillé avec un motif dit en " $N$ » suivant la détermination de $\mathrm{E}$. T. Leeds. Cette pièce appartiendrait à un niveau du début du $\mathrm{II}^{\mathrm{e}} \mathrm{s}$. (après une occupation militaire d'époque augustéenne), qui témoigne du commerce romano-germain.

$2: 2$ A. Tinsixck, Essai sur l'induslrie el les arts dans l'Artois pendant la période gallo-romaine, Paris, 1879, pl. 19, 25.

23 F. Eycics, Lisle des objels recueillis à Sanxay par le R.P. de la Croix, dans (callia, 1944, 2, pp. 98-120, fig. 27, 90, p. 117 (sans trace d'émail).

24 Exemplaires inedits au musée de Treves; voir anssi J. B1:SHE Fox, Fourlh Reporl on the excavalions of the Roman Forl at Richborough, Kent, Oxford, 1949, pl. 41, 192, p. 145. 13. Cuxr.tFFe, Fifth Report on the excavations of the Roman Fort at Richborough, Kent, Oxford, 1968, pl. 38, 130 et 131, p. 96 .

25 Srrabov, IV, 5, 3 ; voir par exemple D. P. S. Psacock, Roman Amphorae in pre-Roman Brilain, dans The Iron Age and ils hillforls. Papers presenled to sir Mortimer Wheeler,
Southampton, 1971; pp. 161-188; B. CuxlfFi, Hengistbury Head, I.ondon, 1978, pp. 38 à 81 ; P. A. TY Yers, Correspondances entre la céramique commune de la Tène III du sud-est de l'Angleterre el du nord de la France, dans Septentrion, 10, 1980, pp. 61-70.

26 Mittelrheinisches Museum de Mayence; Françoise IrNky, op. cil., fig. 12,1, p. 84 .

27 E. T. LEENS, op. cil., fig. 11 ; J. V. S. MEGAw, op. cit., no 263, pp. 156-157.

28 J. V. S. MkGaw, op. cit., no 277, pp. 162-163.

29 G. Ur.Bnat, Die römischen Funde von Bentumersiel, dans Probleme der Küstenforschung im südlichen Noordseegebiel, 11, 1976, pp. 33-65, cf. no 53, pl. IV, pp. 40-41. 

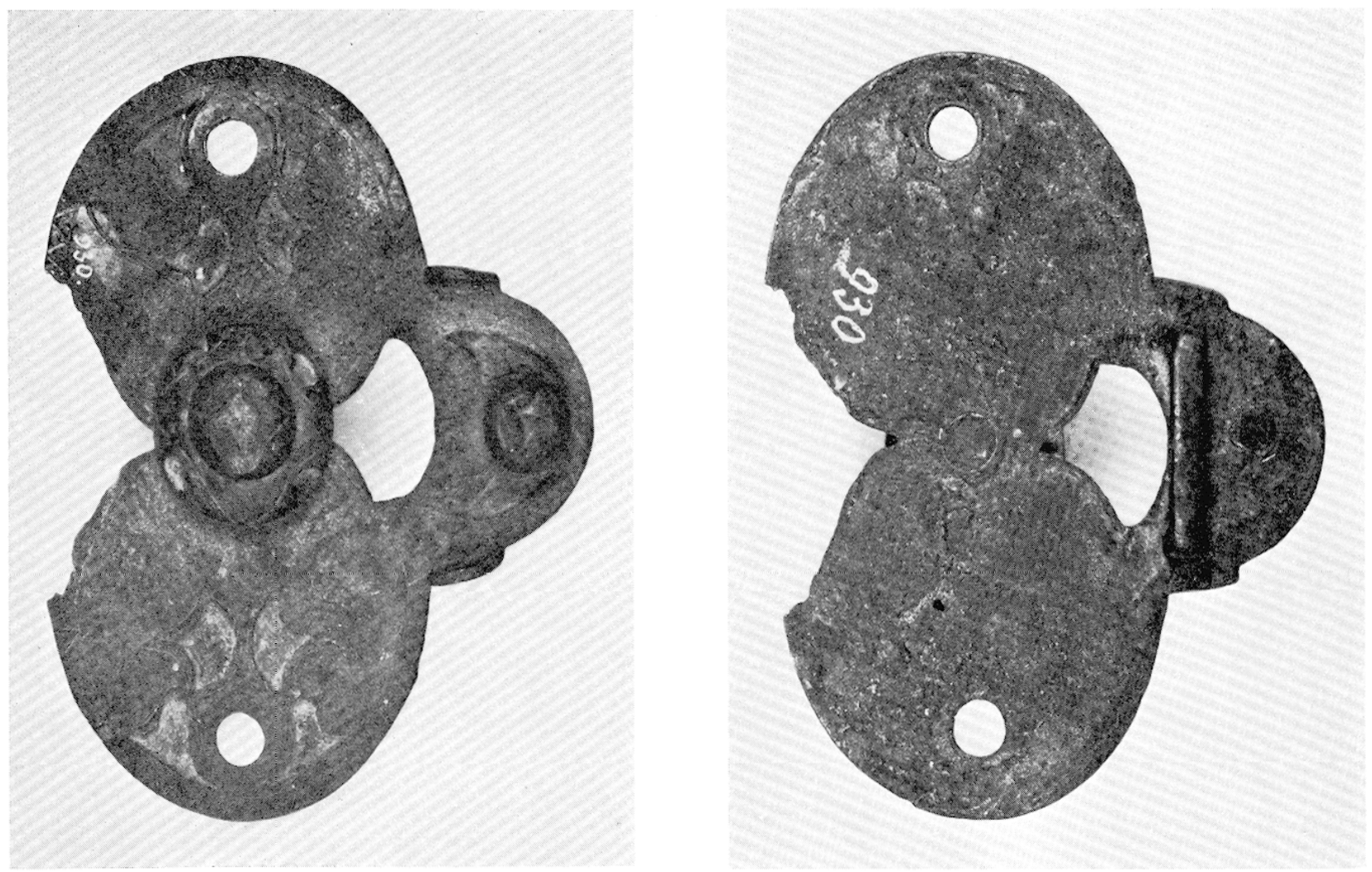

13 Plaque d'Ober Olm, face el revers $(\times 1)$.

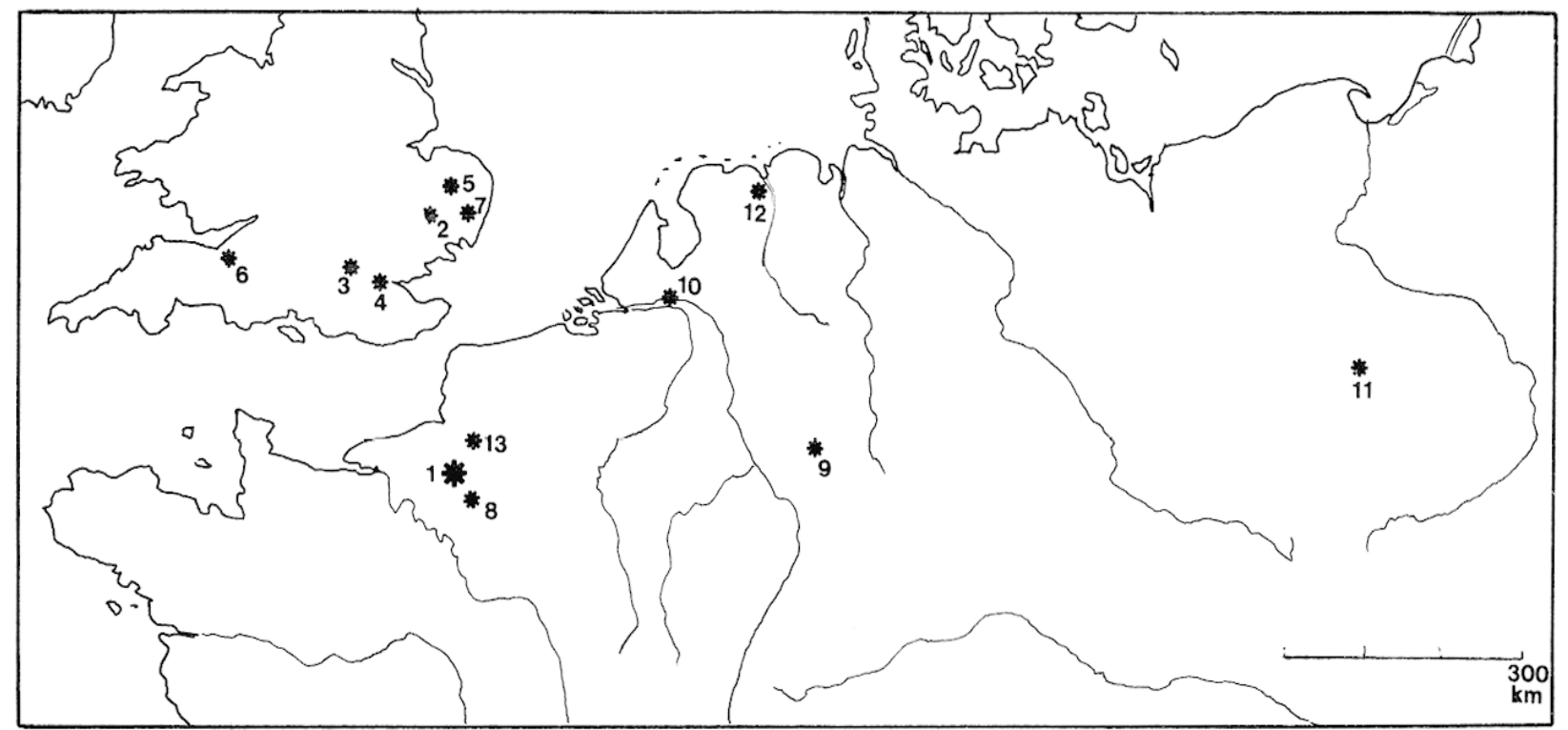

14 Carte de répartition des différents lieux de découvertes mentionnés dans le texte. $1:$ Paillard ; $2:$ Norton ; $3:$ Ilambledon; $4:$ Londres ; 5 : Santon ; 6 : Polden IIill ; 7 : Westhall ; $8:$ Bailleul-sur-Thérain ; $9:$ Ober Olm ; $10:$ Nimègue ; 11 : Leg Pielarshii; 12 : Bentumersiel ; $13:$ Amiens. 
Faut-il accorder une origine commerciale à la découverte de Paillart? Comme pour le miroir de Nimègue, on pourrait songer de préférence au rôle d'auxiliaires belges de l'armée romaine stationnée en Bretagne ${ }^{30}$. Ainsi, la découverte de la patère émaillée d'Amiens, qui porte en inscription quelques sites du mur d'Hadrien, s'explique, pour J. Heurgon, par le passage dans cette ville de troupes faisant route vers la Bretagne, ou en revenant, à moins qu'Amiens n'ait été un séjour pour vétérans ${ }^{31}$. Si, à la différence de la capitale des Ambiens, Paillart ne peut pas avoir été ville étape, elle se situe néanmoins sur le passage obligé de celui qui veut se rendre de Bretagne à Lyon. La fouille du lieu de découverte apporterait peut-être des éléments complémentaires d'explication à la présence de cet objet. En tout cas, il semble bien que la découverte de Paillart, témoigne de l'apogée de l'art celtique insulaire de la fin $d u \mathrm{I}^{\text {er }}$ siècle ou du début $d u \mathrm{II}^{\mathrm{e}}$, ne peut être que le premier élément d'une longue enquête d'un intérêt tout à fait capital pour la compréhension des relations entre le milieu celtique insulaire et le continent (fig. 14) au moment crucial de la conquête de la Bretagne par les Romains.

Germaine Leman-Delerive

C.N.R.S.

N.B. Je voudrais exprimer ici toute ma gratitude à M. le Professeur P.-M. Duval, qui voulut bien relire mon texte, à M. le Professeur V. Kruta pour ses nombreux conseils et ses orientations bibliographiques.

Tous mes remerciements vont aux personnes dont l'amabilité et la générosité ont permis ce travail, en particulier M. G. Dufour, Conservateur du musec de Breteuil, M. Jullien, propriélaire du terrain, M. G. Dilly, Conservateur du musée de Berck, M. J.-Cl. Blanchet, Directeur des Antiquites de picardie. Ce travail n'eùt pas été possible non plus sans l'aide et les autorisations de nombreux archéologues el conservateurs de musér, en particulier Miss Valery Rigby et
Mr. M. Spratling du British Museum, Mrs. E. J. Owles du Moyse Hall Museum à Bury Saint Edmunds, Miss Craster de l'University Museum of Archeology and Anthropology de Cambridge, le Dr M. Egg du Römisch-Germanisches Zentral Museum de Mayence, le $D^{r}$ Decker, du Mittelrheinisches Landesmuseum de Mayence. Mr. B. Raftery, de l'Université de Dublin, et Mr. J. J. Wymer, Secrétaire du Suffolk Institute of Archeology, me furent également d'un grand secours. Je dois igalement de précieux renseignements à $A$. CahenDelhaye, du Service National Belge des Fouilles, au $D^{r}$ H. Cüppers, du Landesmuseum de Trèves, à M. M. Feugère (C..N.R.S.), à propos des pièces romaines.
30 A l'inverse du cas hollandais, on ne connaît pas jusqu'ici de corps d'auxiliaires levé en pays bellovaque, quoique d'autres Belges aient fourni des troupes qui stationnèrent en Bretagne (voir R.E., s.v. Cohors, IV, 1901, col. 316, 317 , exemples des Ménapiens, des Morins et des Nerviens!. Quant à l'utilisation, ou à la récupération d'armes indigènes par l'armée romaine, on peut rappeler les pièces de Newstead : J. Curle, A Roman Frontier Post and its Peole: the Fort of Newstead in the Parish of Melrose, Glasgow, 1911.

31 J. Helrgox, La palère d'Amiens, dans Fondalion E. Piot, Monuments el Mémoires publics par l'Académie des Inscriplions et Belles-Letlres, XI.VI, 1952, pp. 93-115. 


\author{
ANNEXE I \\ Analyse des f́mat: \\ par Jacques Bonte*
}

\title{
Moyens utilisés :
}

1. Microsonde de Caastaing du Microscope Électronique à Balayage (Jcol) de l'Institut des Sciences des Matériaux; tension 25 et $35 \mathrm{kw}$, analyseur à cristaux LIF, PE'T, RAP.

2. Goniomètre de diffraction des rayons X CGR (Eudil)*, avec anticalhode Cu et Co.

3. Dépouillement des spectres sur ordinateur Solar 16 (Eudil).

\section{Résultats :}

\section{A) Émail verl.}

C'est une couverte plus ou moins transparente et de faible épaisseur $(<1 \mathrm{~mm})$ à la périphérie de la plaque. Cette couverte a d'ailleurs mouillé partiellement le revers de l'objet lors de la fusion.

Les éléments chimiques présents qui ont été détectés sont: $\mathrm{Si}, \mathrm{Pb}, \mathrm{Cu}, \mathrm{Ba}, \mathrm{Na}, \mathrm{Sn}$.

La méthode utilisée (microsonde électronique) ne permet pas de préciser, sans étalonnage préalable sur des substances identiques, la nature des composés chimiques présents ainsi que leur tencur.

D'autre part, les ćléments légers comme l'Oxygène, le Bore, et le Fluor ne pouvaient être détectés avec l'équipement utilisé lors de l'analyse; ceci ne permet pas de savoir si des fondants comme $\mathrm{B}_{2} \mathrm{O}_{3}$ et $\mathrm{Na}_{2} \mathrm{~F}_{6} \mathrm{Si}$ ont été utilisés pour faciliter la fusion de l'émail et le mouillage du métal par le verre.

Néanmoins, on peut dire que l'émail vert est un "verre" alcalin au Plomb, coloré en vert par des ions cuivre à l'état d'oxydation correspondant à l'oxyde CuO. Ces ions cuivre proviennent vraisemblablement de la plaque par suite d'une réaction entre l'oxyde couvrant la plaque et l'émail pendant la fusion. Ceci est conforme aux diverses observations rapportées par J. D. Bateson ${ }^{32}$.

\footnotetext{
- Ecole Eniversitaire d'Ingenieurs de Lille (E.L.L.I.I.. lépartement des Sciences des Materiaux (D.S.M.?.
}

32 J. 1). BAтtsox, Enamel uorking in iron aye, Roman and sub-Roman Brilain. The producls and lechnics; British Archeological Reports, Oxford, 1981, p. 72 et 75 . 
B) Émail jaune.

C'est un "verre" opaque.

La matrice verre est un silicate de calcium et de baryum avec des traces de Cerium.

On trouve une forte proportion de Plomb.

Ce plomb joue un double rôle : fondant diminuant, comme pour l'émail vert, la température de "fusion " et améliorant la mouillabilité de la matrice silico-alcaline; colorant sous forme d'oxyde (voir plus loin) et sous forme de composé chimique avec l'antimoine.

Les autres éléments identifiés à la microsonde sont : le Mo, Fe, Cd, Sn et Cu (ces éléments jouant le rôle de colorants); l'Antimoine et l'Étain accompagnés de traces de Titane jouant le rôle d'opacifiants.

La diffraction des rayons $\mathrm{X}$ a permis d'identifier, entre autres des substances cristallisées dans la matrice "verre ", elle-même non cristalline, deux composés : la litharge, $\mathrm{PbO}$ (réseau hexagonal) et l'orthoantimonate de plomb $\left(\mathrm{Pb}_{3}\left(\mathrm{SbO}_{4}\right)_{2}\right)$.

Ces deux substances sont bien connues comme colorant jaune dans les émaux de cette époque ${ }^{33}$.

Nous attribuons à l'Antimoine un double rôle : celui de colorant comme ci-dessus, et d'opacifiant vraisemblablement à l'état d'antimonate double de sodium et de calcium, et ceci conformément à la littérature relative aux émaux anciens ${ }^{34}$, aux émaux et verres modernes ${ }^{35}$.

\section{G) Émail rouge.}

C'est ici encore un "verre" opaque. La matrice verre est à nouveau un silicate de baryum, de calcium et de strontium avec des traces de Cerium.

On retrouve la même forte proportion de plomb que dans les deux autres émaux.

Les opacifiants sont ici aussi les oxydes de $\mathrm{Sb}, \mathrm{Sn}, \mathrm{Bi}$ et $\mathrm{Zn}$.

Les colorants sont à base d'oxyde de fer, de cuivre, de plomb, de cadmium et de magnésium.

Des traces d'Or et d'Argent ont été également détectées.

La présence de $\mathrm{Mg}$ et de très faibles quantités de Soufre suggère que les fabricunts de l'émail ont utilisé une argile dolomitique comme une des matières premières ainsi que Sayre le signale ${ }^{36}$.

Par diffraction de rayons $X$, on a identifié comme substances cristallines présentes dans l'émail rouge, de la cuprite, $\mathrm{Cu}_{2} \mathrm{O}$ (cubique) et aussi de l'hématite, $\mathrm{Fe}_{2} \mathrm{O}_{3}$ (hexagonale).

Cette dernière substance est en très faible quantité et apparemment rarement utilisée comme colorant à cette époque ${ }^{37}$.

Il faut noter que l'oxyde $\mathrm{Cu}_{2} \mathrm{O}$ est la variété la moins oxydée du Cuivre que l'on trouve dans les trois émaux, ce qui suggère que la fusion de cet émail s'est faite en milieu non oxydant, ou réducteur (voir conclusion).

Ajoutons que les substances cristallines identifiées par diffraction des RX n'existent que dans les "verres " rouge et jaune qui sont aussi les verres ou émaux les plus épais. Ceci peut provenir soit de cristallisation pendant la période de refroidissement, au sein de la matrice verre, mais plus vraisemblablement de particules solides non "dissoutes» dans le verre silico-alcalin.

Ces particules sont vraisemblablement des poudres colorées ajoutées au verre par l'artiste avant

33 J. I). BATESON, op. cit., p. 70-71.

34 Ibid.

35 W. D. KiNGERY, H. K. BOWEX and D. R. UHLMAXN, Inlroduclion to ceramics, p. 669-673; W. A. WEYL, Coloured Glasses, dans Sociely of Glass Technology, Sheffield, 1951.
36 E. V. SAYRe, Summary of the Broolihaven Programm of Analysis of Ancient Glass, Applicalion of science in the examination of Works of Arl, Boston, 1965, p. 146 à 164.

37 J. D. BATESON, op. cit. 
la fusion de l'émail soit pour augmenter la coloration, soit, plus sûrement, pour obtenir un verre opaque masquant bien la coloration verte qui pourrait provenir de la réaction intempestive mais inévitable entre l'oxyde recouvrant la plaque et la matrice "verre".

\section{Conclusions :}

D'après les compositions qualitatives des trois émaux, et d'après la littérature relative aux émaux anciens et modernes, il est raisonnable de penser que : la matrice verre est un silicate complexe d'alcalin $(\mathrm{Na})$, d'alcalino-terreux $(\mathrm{Ba})$ et de Plomb; la température de fusion d'un tel verre est comprise entre 800 et $850{ }^{\circ} \mathrm{C}$ mais a pu être abaissée très fortement par l'utilisation de fondants (anhydride borique, fluorure de sodium, etc.) (nous n'avons aucunc preuve expérimentale de ce fait); les colorations sont obtenues par la dispersion dans la matrice "verre" de pigments minéraux naturels restant inattaqués par le silicate, ou par leur dissolution et réaction avec la matrice. La coloration verte résulte, clle, d'une réaction du "verre " transparent avec la plaque ou alliage de cuivre; il est vraisemblable que l'émail vert est le reste d'une couverte transparente qui recouvrait toute la pièce mais qui a disparu, au droit des zones rouges et jaunes, soit par abrasion mécanique (volontaire ou non, usure) soit par faïençage et éclatement dû à une mauvaise adhésion sur les émaux rouge et jaune lors du refroidissement consécutif au deuxième émaillage transparent, celui-ci effectué dans des conditions différentes de durée, de température et d'atmosphère. (Cette dernière hypothèse n'a pu être étayéc de façon certaine lors de l'étude trop rapide des émaux recouvrant la plaque).

Quant à la technologie utiliséc, on peut écrire qu'elle n'était ni complexe ni difficile à mettre en œuvre. Les émaux ont pu être produits par les dépôts de poudres broyées et mélangées puis cuites au four en atmosphère plutôt réductrice (charbon de bois) avec fusion partielle pour les émaux rouges et jaunes (ce qui les a laissés opaques) et fusion totale de la couverte transparente qui s'est colorée en vert par réaction avec la plaque de cuivre lors d'une deuxième cuisson, cette fois-ci en atmosphère oxydante (four ouvert).

\section{Remarques :}

Il serait intéressant et utile de pouvoir faire l'analyse quantitative de ces émaux (mais celle-ci serait destructricc) et de rechercher et doser les éléments légers $F, B, O$, pour mieux connaitre la technologie utilisée (température de cuisson en particulier).

Un examen micrographique de coupes polies des zones émaillées renseignerait encore davantage sur la technique d'émaillage utilisée mais cette dernière observation serait très agressive et destructrice pour l'objet.

J. B. 


\author{
ANNEXE II \\ L'emplor du compas lors de la conception et de la réalisation de la Plaque de Paillart \\ par Venceslas Kruta
}

La plaque émaillée de Paillart a été réalisée à partir d'un schéma initial simple, connu par les artisans celtiques depuis leurs premières tentatives d'utilisation systématique du compas, au ve $\mathrm{s}$. av. J.-C. : quatre cercles sont distribués sur les branches de deux axes perpendiculaires; ils s'entrecroisent dans la partie centrale et dessinent à l'extérieur une rosace quadrilobée ${ }^{38}$. La variante utilisée ici ne peut toutefois être inscrite dans un cercle imaginaire, car les centres des quatre cercles ne sont pas disposés à même distance du point de croisement des axes et leurs diamètres ne sont pas identiques : les centres (A) des deux plus grands cercles (a) sont placés sur l'axe, supposé horizontal, qui correspond à la ligne de passage de la courroie sur le revers, de sorte que leur distance du point de croisement central soit inférieure d'environ un quart à leur rayon; ils se croisent ainsi sur l'axe orthogonal (vertical) et fournissent l'ossature de la partie centrale de la composition (fig. 15). Deux autres cercles plus petits (b) ont leurs centres (B) placés de part et d'autre du point central sur l'axe vertical, à une distance qui est cette fois légèrement supérieure à leur rayon; ils ne se croisent donc pas ni ne se touchent et la forme extérieure que dessinent les deux paires de cercles présente un axe long (vertical) et un axe court (horizontal) ${ }^{39}$.

Quelques imprécisions ont eu pour conséquence l'altération de la disposition régulière du schéma initial et ont conditionné la mise en place de l'ornementation intérieure. La plus importante est le léger infléchissement de l'axe vertical au point où il croise l'axe horizontal : ainsi, au lieu d'être parfaitement rectiligne, il forme d'un côté un angle obtus d'environ $175^{\circ}$ (fig. 15 , vers la gauche).

L'ornementation intérieure est construite à partir de cercles qui correspondent à des subdivisions des précédents : les cercles moyens $c$ (centres C), utilisés notamment pour tracer les grands triangles curvilignes ajourés de la partie centrale qui se trouvent sur l'axe horizontal, ont un rayon égal au tiers du rayon des cercles $a$; le rayon des petits cercles $d$ (centres D) employés pour l'élaboration de tous les autres ajours de cette forme, est le quart du rayon du cercle $b$.

La remarquable régularité des rayons de tous les cercles qui servirent à élaborer le schéma initial, déterminant pour la mise en place des ajours et des alvéoles destinés à contenir l'émail, est peut-être la conséquence de l'emploi de calibres ou de plusieurs compas à écartement fixe (fig. 16).

$38 \mathrm{Cf}$. notamment la construction des disques ajourés de Somme-Bionne et de Cuperly : M. Lenerz DF Wildse, Zirkelornamentik in der Kunst der Latènezeil, München, 1977, pl. $10,21$.
39 Une construction de ce type serait à l'origine d'une des plaques de Kleinaspergle : ibid., pl. 13 et 35/2. 


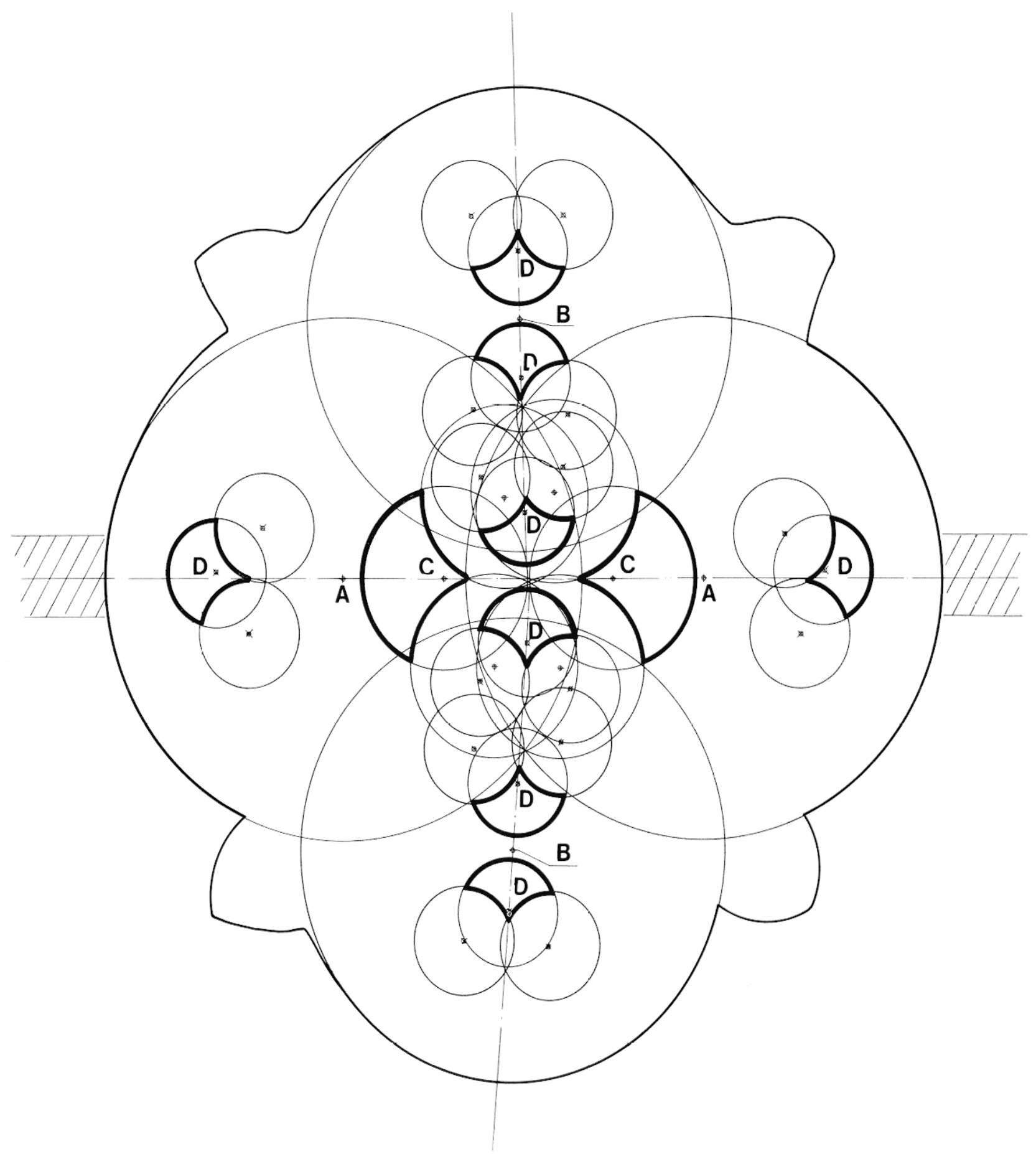

15 Schéma de la construction géométrique de la plaque de Paillart : un assemblage de cercles de quatre diamètres différents (centres $\mathrm{A}, \mathrm{B}, \mathrm{C}$ et $\mathrm{D}$ ), ordonnés suivant deux axes perpendiculaires la partie inférieure de l'axe vertical présente au point de croisement, un infléchissement vers la gauche de $5^{\circ}$ ), détermine l'emplacement des ajours (traits épais) et la forme du contour extéricur. Les mêmes procédés furent employés pour les alvẻoles destinés à contenir l'émail (non figurés ici) qui étaient également prévus avant la fusion de la plaque. L'existence d'une épure préalable, complète et suffisamment détaillée, constitue une prémisse indispensable à la réalisation. 

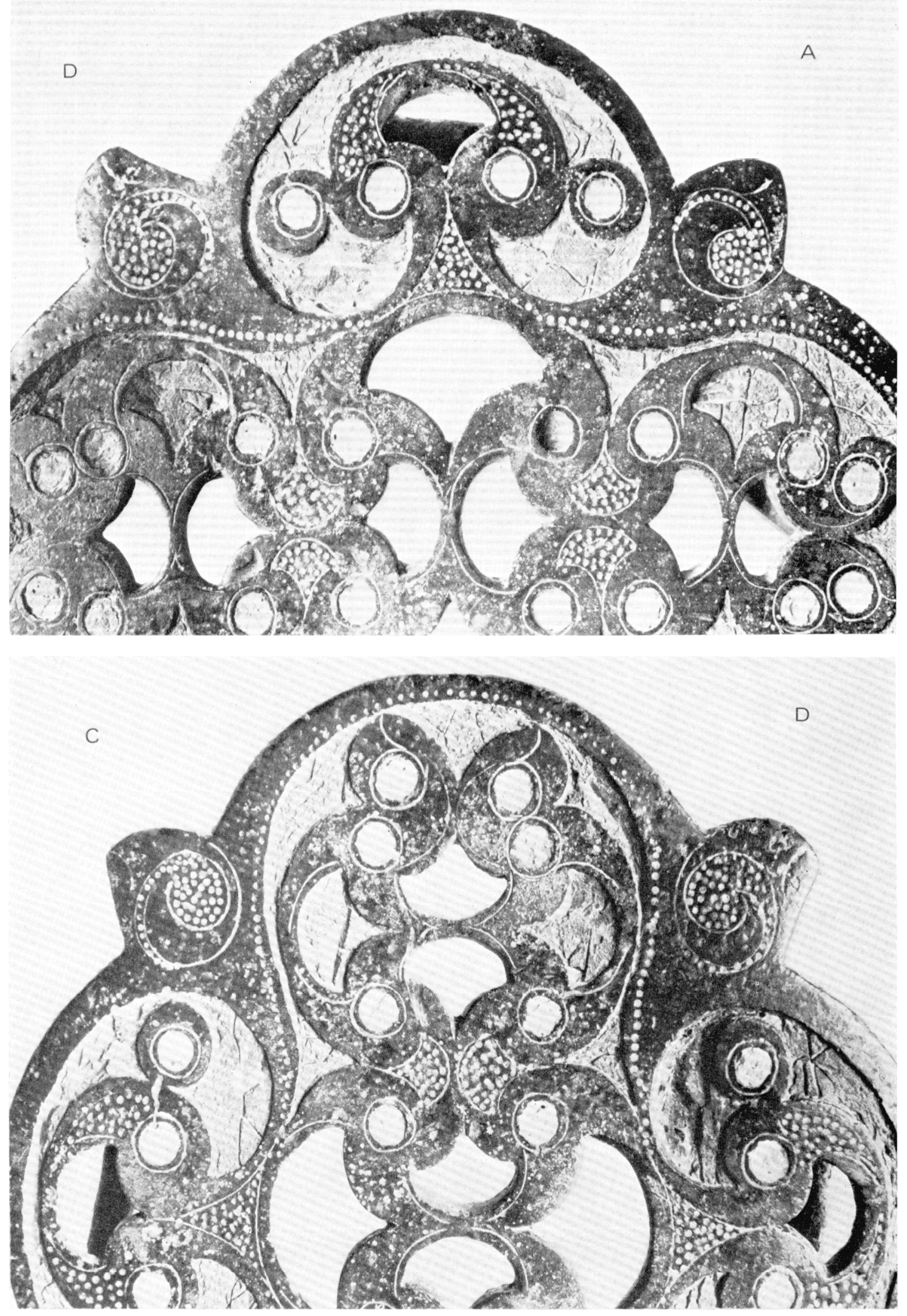

16 Deux détails de la plaque de Paillart, montrant les parties gravées après la fonte à l'aide d'un compas $(\times 1,70)$. 

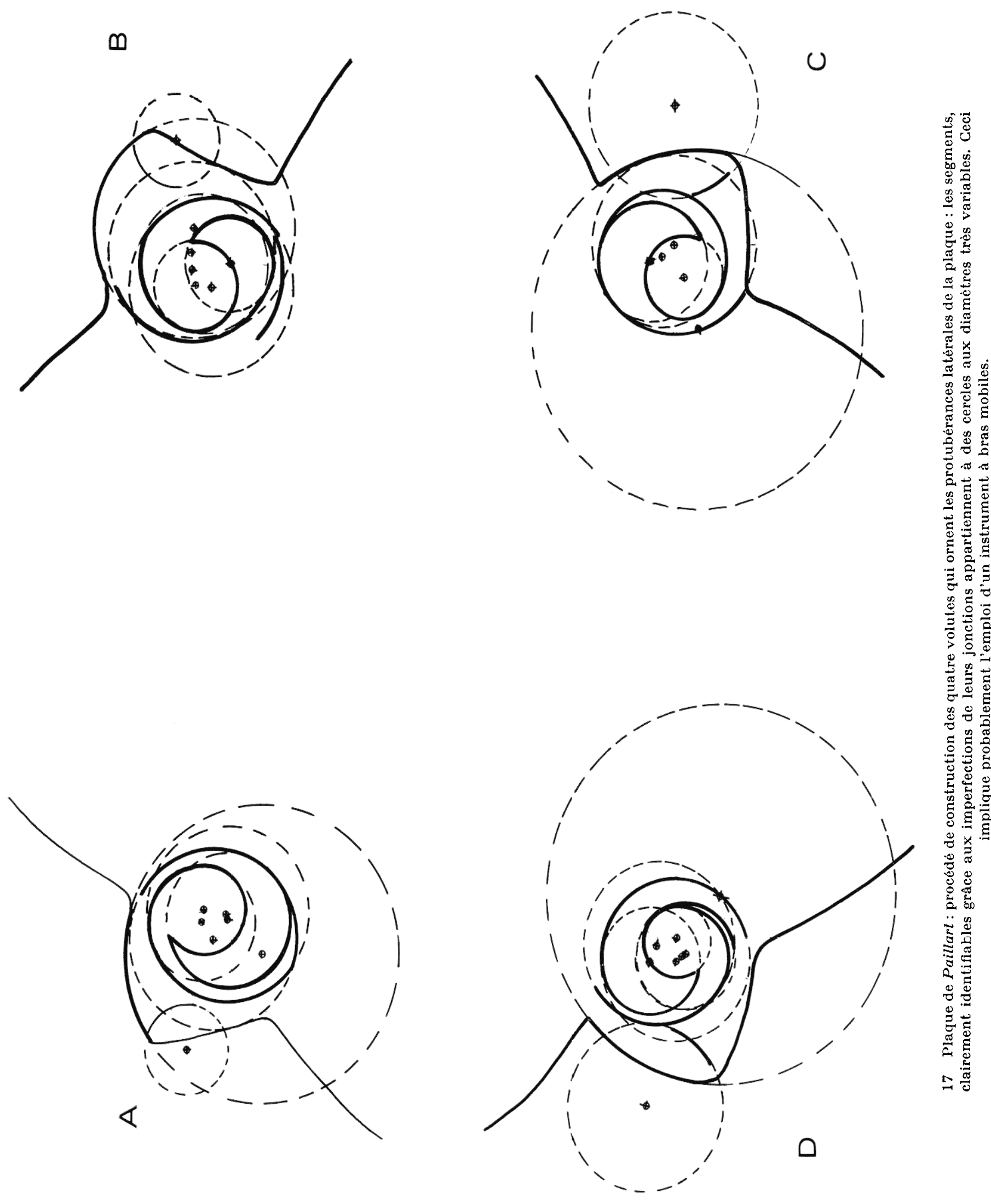
C'est, au contraire, probablement un compas à écartement variable qui a été utilisé pour la réalisation du décor gravé qui complète et souligne les éléments émaillés et ajourés. L'examen comparatif des volutes qui ornent les quatre protubérances latérales de la plaque (fig. 17) indique en effet que, tandis que le procédé de construction à partir de plusieurs segments de cercle reste le même, les diamètres présentent des variations importantes. Toutefois, comme les centres des cercles se confondent avec le remplissage de points, on ne peut totalement exclure l'utilisation d'un module au contour hélicoïdal. Fait important, certaines lignes ont été gravées directement en profondeur par l'un ou l'autre de ces procédés, d'autres seulement tracées très légèrement et reprises à la main. C'est notamment le cas des lignes pointillées. Ce genre de travail au compas est tout à fait comparable à celui qui a été observé sur les miroirs britanniques ${ }^{40}$. Le tracé très irrégulier des cercles qui entourent les alvéoles circulaires remplis d'émail jaune indique qu'ils ont été rẻalisés entièrement à main levée, sans gravure préalable au compas. La raison en est probablement le fait que la pointe de l'instrument aurait dû s'appuyer sur l'émail et aurait pu l'endommager.

En conclusion, il apparaît comme certain que l'ornementation de la plaque de Paillart n'a pu être réalisée qu'à partir d'une ébauche préalable, élaborée au compas en suivant des règles relativement simples dont l'application est attestée sur différents objets celtiques depuis le ve $\mathbf{s}$. av. J.-C. Ces constructions au compas connurent une vogue particulière dans l'art insulaire des premiers siècles av. et ap. J.-C. et donnent aux objets ainsi conçus et décorés une unité qui vient peut-être plus de l'emploi de démarches analogues que de relations directes entre les différentes œuvres. Les ressemblances qu'ils présentent seraient donc structurelles et ne reflèrent donc pas nécessairement l'origine commune d'un atelier déterminé ou son influence.

V. K.

N.B. Les photographies de cet article sont l'œuvre de M. H. Josse (fig. 3), de M me Leman (fig. 6), de M. V. Kruta (fig. 4 et 16) et de M. H. Thönig du Landesmuseum de Trier (fig. 12). Celles des autres plaques nous ont été communiquées par le British Museum (fig. 5), le Mittelrheinisches Landesmuseum de Mayence (fig. 13). Les dessins et relevés ont été exécutés par R. Pengelly du British Museum (fig. 7, $1^{\prime}$ et $2^{\prime}$ ) avant le départ du no 1 dans la collection Menil de Houston (Texas) et par F. Lagarde (fig. 15 et 17).

40 G. P. Lowery et R. Savage, Cellic Design with Compasses as seen on the Holcombe Mirror, dans P.-M. Duval et C. F. C. Hawkes, Cellic Art in Ancient Europe. Five
Protohistoric Centuries - L'Art celtique en Europe protohistorique: débuts, développements, sigles, techniques, LondonNew York San Francisco, 1976, pp. 219-231. 